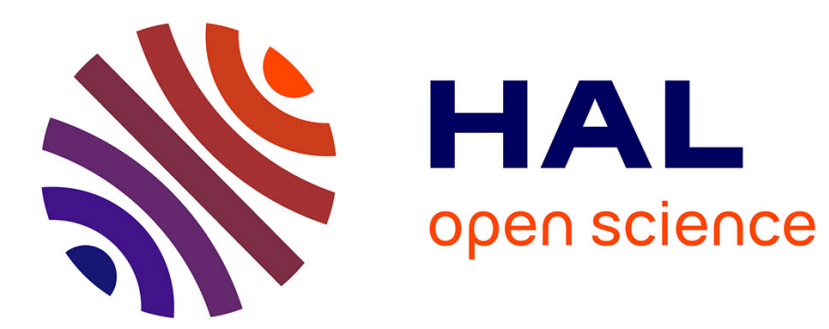

\title{
Les emprunts du grec à l'égyptien
}

Jean-Luc Fournet

\section{To cite this version:}

Jean-Luc Fournet. Les emprunts du grec à l'égyptien. Bulletin de la Société de Linguistique de Paris, 1989, LXXXIV, 1, p. 55-80. halshs-00001023

\section{HAL Id: halshs-00001023 https://shs.hal.science/halshs-00001023}

Submitted on 13 Jan 2004

HAL is a multi-disciplinary open access archive for the deposit and dissemination of scientific research documents, whether they are published or not. The documents may come from teaching and research institutions in France or abroad, or from public or private research centers.
L'archive ouverte pluridisciplinaire $\mathbf{H A L}$, est destinée au dépôt et à la diffusion de documents scientifiques de niveau recherche, publiés ou non, émanant des établissements d'enseignement et de recherche français ou étrangers, des laboratoires publics ou privés. 
Bulletin de la Société de linguistique de Paris,LXXXIV 1, 1989

\section{LES EMPRUNTS DU GREC À L'ÉGYPTIEN}

Sommarre. - Nous dressons tout d'abord un inventaire des emprunts du grec à l'égyptien en respectant les différents niveaux de langue en fonction de leur acclimatation dans la langue emprunteuse (emprunts parfairement assimilés, pérégrinismes et termes du grec d'Égypte et de la Septante). On constate, dans un second temps, par une analyse des motivations et des modalités (processus d'introduction et d'adaptation) de ces emprunts, entre autres, le conservatisme du grec qui n'a recours à des mots étrangers que pressé par la nécessité, ainsi que la concordance entre les résultats de cette étude et les données historiques et littéraires.

Si le rôle qu'a exercé l'Égypte dans l'inconscient collectif des Grecs a été depuis longtemps souligné, si les relations entre ces deux peuples ont fait l'objet de recherches, l'influence de l'Égypte sur la Grèce n'a été que trop succinctement étudiée à travers le prisme révélateur du langage. Le passage de mots d'une langue dans une autre est pourtant un signe objectif d'une relation privilégiée et subjective ${ }^{1}$.

1. La bibliographie (à laquelle nous renverrons de façon abrégée) est non seulement réduite mais, pour une part, vieillie :

P.-E. JABlonsky, Opuscula, I (édité avec supplément par Te Water, Leyde 1804).

A. Wiedemann, Sammlung altägyptischer Wörter, welche von klassischen Autoren umschrieben oder übersetzl worden sind, Leipzig 1883.

A. Erman, "Aegyptische Lehnworte in Griechischen", Beiträge zur Kunde der indo-germanischen Sprachen, 7 (1883), p. 336-8.

W. SpIEgelberg, "Aegyptische Lehnworte in der älteren griechischen Sprache», KZ 41 (1907), p. 127-132.

G. Nencioni, "Innovazioni africane nel lessico latino», SIFC 16 (1939), p. 8-23.

B. Hemmerdinger, "Noms communs grecs d'origine égyptienne", Glotla, 46 (1968), p. 247-254. 238-247

A. G. Mac Gready, "Egyptian Words in the Greek Vocabulary", ibid., p. 38 247. $247-254$

R. H. Pierce, "Egyptian loan-words in ancient Greek", Symbolae Osloenses, 46 (1971), p. 96-107.

Faut-il citer l'ouvrage en russe de P. V. Fernštedt, Mots d'emprunt égyptiens 
Nous nous proposons d'étudier ici les emprunts de noms communs grecs à la langue égyptienne. La langue de départ est bien évidemment l'égyptien proprement dit, parlé à l'époque pharaonique, incluant le démotique (dém.), mais aussi le copte ${ }^{2}$.

Nous avons résolument écarté, outre les hypothèses indémontrables, toutes les transcriptions de noms de plante livrées par Dioscoride et le botaniste Apulée, pour lesquelles il est souvent impossible de trouver un correspondant satisfaisant, ainsi que certaines étymologies fausses qui persistent encore aujourd'hui ${ }^{3}$.

\section{INVENTAIRE}

\section{Emprunts certains.}

Le critère de classification en fonction du plus ou moins grand degré de pénétration dans la langue nous a semblé, dans un premier temps, le plus pertinent. Apparaissent ainsi différents groupes d'acclimatation. Le premier (A) englobe les emprunts parfaitement assimilés par le grec, bénéficiant donc d'une totale adaptation morphologique (suffixe hellénisant) et d'une capacité à produire dérivés et composés. Le second groupe (B) rassemble les "mots-transcriptions» ou pérégrinismes (all. Fremdwörter par opposition aux Lehnwörter). Ce sont des vocables sentis comme étrangers, le plus souvent des transpositions sans aucune vivacité dans la langue emprunteuse, rares, non-productifs, souvent indéclinables et soulignés dans les textes par certaines

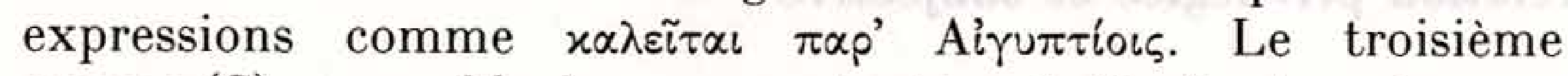
groupe $(\mathrm{C})$ rassemble les emprunts bien intégrés dans le grec parlé en Égypte à partir de la période ptolémaïque. Implantés

au grec, Moscou 1953, illustrant le "pan-égyptianisme» fantaisiste dans tout son excès?

Enfin, je tiens à remercier de leurs conseils M. Olivier Masson, à l'origine de cette recherche et M. Laurent Dubois, ainsi que M. Jean Yoyotte qui a contrôlé et enrichi l'information égyptologique de cet article.

2. Pour l'égyptien, nous renvoyons à Erman-Grapow, Wörterbuch der ägyptischen Sprache, 1926-1971 (=Wb). Pour le démotique, à W. Erichsen, Demotisches Glossar, 1954 (=E). Pour le copte, à Crum, Coptic Dictionary, Oxford 1939 et à W. Vycichl. Dictionnaire étymologique de la langue copte, 1983. Pour la phonétique égyptienne, à J. Vergote, Phonétique historique de l'égyptien, Louvain 1945. Nous utilisons les abréviations suivantes pour les dialectes coptes : $\mathrm{B}=$ Bohaïrique, $\mathrm{A}=\mathrm{Akhmîmique}, \mathrm{S}=$ Sa îdique, $\mathrm{F}=$ Fayoumique. Les abréviations des papyrus sont celles du $L S J$.

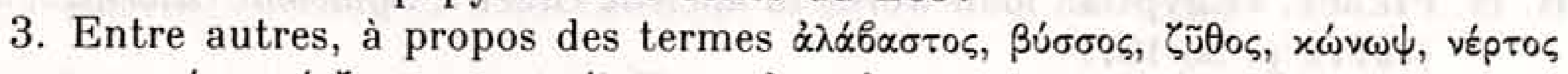
$\xi i \varphi \circ \varsigma, \sigma u p \mu \alpha i \alpha, \sigma \varphi i ́ \xi \xi$, pour ne citer que les plus récentes et les plus tenaces. 
au milieu d'une civilisation étrangère, en confrontation avec des réalités non-helléniques, les Grecs ont dû avoir recours à des mots qui, s'ils constituent des transcriptions, n'en sont pas moins bien vivants et productifs. Il faut y ajouter certains termes utilisés dans la Septante, qui fut rédigée en Égypte.

\section{A. Mots assimilés par le grec.}

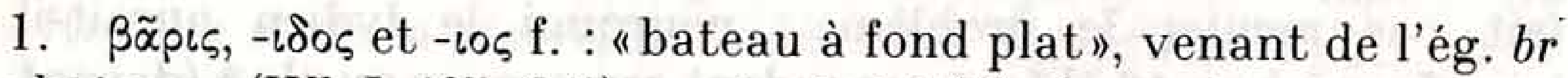
"bateau" (Wb I 465, 8-9), copte bairi (B). Le mot est passé en latin sous la forme barca (fr. barque). Morphologiquement assimilé, il n'en a pas moins gardé la marque de son origine. Sa première occurrence figure dans Les Suppliantes d'Eschyle (probablement 463), où il est employé dans un contexte

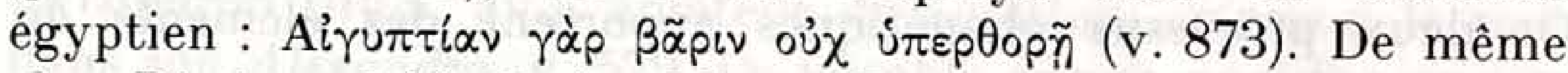
chez Diodore, I 96, 8; Jamblique, De mysteriis, 6, 5; Plutarque, Mor. 358 A; Themistius, Orationes, IV 49. Mais le terme en arrive à perdre sa couleur exotique : Lycophron (747) l'emploie pour qualifier le radeau d'Ulysse. De plus le mot a fourni des composés, témoignages de son assimilation: $\beta \alpha p^{\prime} i \alpha_{\alpha}$ (Soph. Frg. 517), "celui qui va dans un bateau", ßoub́ápas (Hérodien,

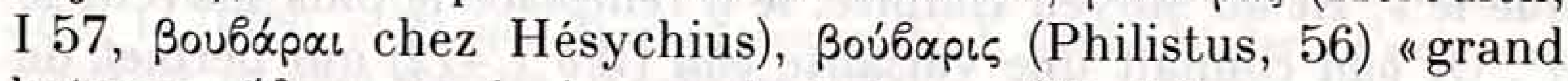
bateau", «’bapıs "qui n'a pas de bateau» (Hsch.).

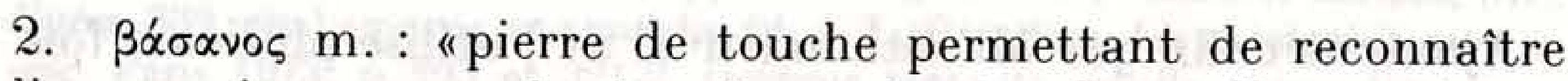
l'or", puis "usage de la pierre de touche», d'où "mise à l'épreuve", ayant donné naissance à toute une série de composés et dérivés du registre judiciaire. Première occurrence : Théognis, 417.

Sethe ${ }^{4}$, le premier, a proposé de faire remonter le mot à l'ég. $b h n$ (Wb I 471) qui désigne, entre autres, le schiste provenant du Wadi Hammâmat dont la dureté permettait qu'on l'utilisât comme pierre de touche. Les auteurs classiques ont bien cherché l'origine de $\beta \alpha \sigma \alpha v_{i}^{\prime} \tau s$ sitos, latin basanites en Égypte : Invenit eadem Aegyptus in Aethiopia quem vocant basaniten, ferrei coloris et duritiae, unde ei nomen dedit (Pline, XXXVI 58). Ptolémée,

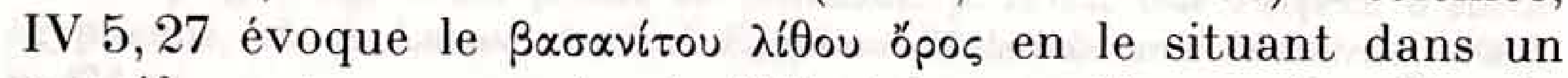
massif montagneux entre le Nil et la mer Rouge. Or, d'après Sethe, bhhn $\left(=^{*}\right.$ bah̆an $)$ en même temps qu'il a donné l'hébreu bachan "examiner (spécialement pour les métaux)", a dû entrer 
dans la langue des Hittites ou d'un autre peuple d'Asie Mineure, se transformant en bašan, forme sous laquelle il serait passé en

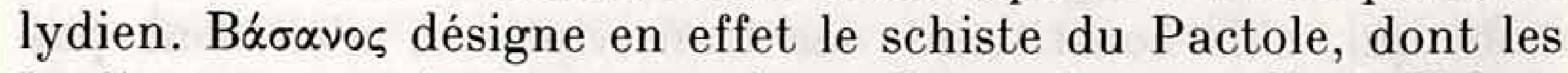
Lydiens se servaient comme pierre de touche pour l'or ${ }^{5}$. Il faut bien reconnaître, avec Kretschmer, que le passage de $h$ à $s$ paraît "quelque peu mystérieux». Le h égyptien se rend en grec par $x$ ou $\chi$. Recourir à l'explication d'un intermédiaire lydien ne fait que reculer le problème: pourquoi le lydien aurait-il transformé $h$ en $s$ ? Il faut cependant remarquer que le $h$ (devant une voyelle de timbre $a$, ce qui est ici le cas) se transcrit en copte, sauf en akhmîmique, par la lettre $s$. Cet argument n'est valable que si le phénomène existait au moins avant le $\mathrm{VI}^{\mathrm{e}} \mathrm{s}$. (date approximative de la première attestation du mot en grec). Or deux processus phonétiques apportent des éléments de réponse :

- Déjà au Nouvel Empire, le $h$ subit une prépalatisation qui le transforme en un yod sourd $|\xi|$ dans la plupart des mots ${ }^{6}$.

- Dans les textes des pyramides, le $\underline{h}$ et le $s$ permutent souvent l'un avec l'autre, le $\underline{h}$ représentant un état plus récent que le $s$. Et au Moyen Empire, le $h$ remplace le plus souvent le $\underline{h}^{7}$. Or $b h \underline{h}$ connaît une variante $b \underline{h} n$ (Wb I 472) qui permet de reconstruire une forme ${ }^{*} b s ̌ n$ des plus plausibles.

Ajoutons enfin que Manéthon (III $\mathrm{s}$. av.) utilise plusieurs fois le sigma pour rendre un $h^{8}$.

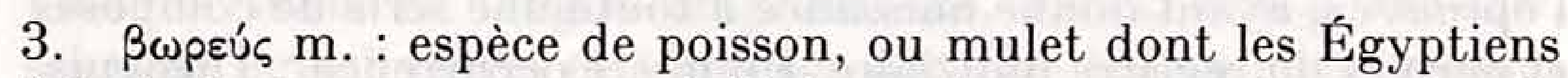

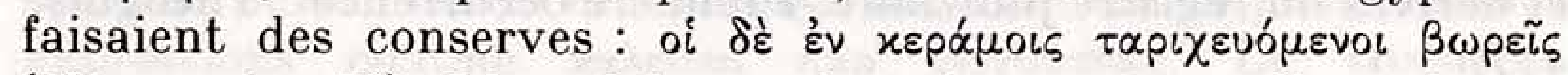
(Xénocrate, 76). L'emploi, par le même auteur, d'une forme $\beta \omega p i \delta i o v$ "conserve de $\beta \omega \rho \varepsilon u ́ s$ ", reprise en $\beta o u p i \delta \iota \alpha$ chez Alexandre de Tralles, nous montre que le vocable était passé, sinon dans la langue courante, du moins dans un domaine spécialisé ${ }^{9}$. Le mot remonte à l'ég. $b r$ (Wb I 465, 20), copte bōre (S) «mulet» (Mugil Cephalus) et il est encore utilisé par les pêcheurs égyptiens sous

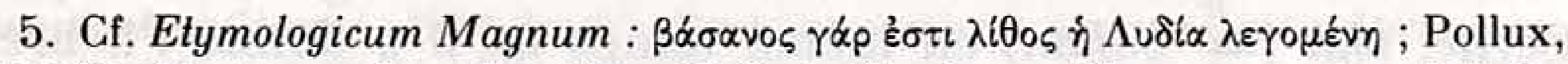

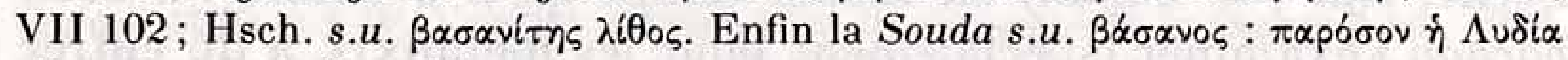

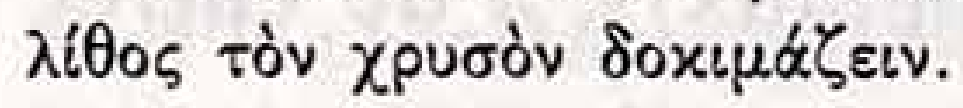

6. Vergote, Phonétique, p. 65.

7. Vergote, o.c., p. 66.

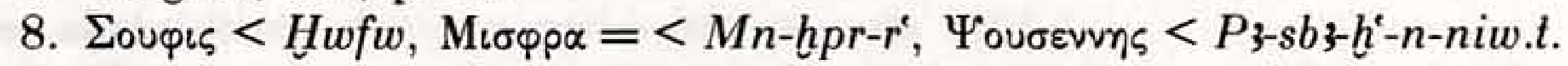

9. Xénocrate ( $\mathrm{I}^{\mathrm{er}}$ ap.) et Alexandre de Tralles (vie ap.) sont tous deux médecins. 
la forme arabe $b \bar{u} r \bar{l}^{10}$. Le terme a été caractérisé par le suffixe -eús bien attesté pour des noms de poissons, en particulier de mulets et de muges ${ }^{11}$.

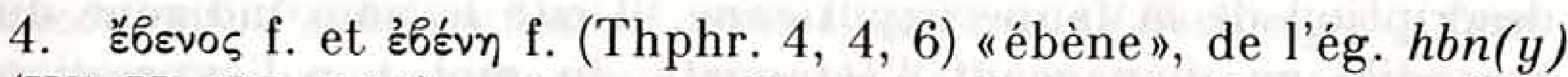
(Wb II 487, 7-12), même sens ${ }^{12}$. Attesté depuis Hérodote (III, $97,114)$ il a donné naissance à plusieurs composés. L'Égypte importait l'ébène (Dalbergia Melanoxylon) de Nubie, du "Kouch" et de la "terre de Pount» des Égyptiens. Peut-être le mot est-il d'origine nubienne ${ }^{13}$, en tout cas d'origine africaine.

5. Ép $\pi \iota \varsigma$ m. : "sorte de vin". Le mot se trouve chez Hipponax,

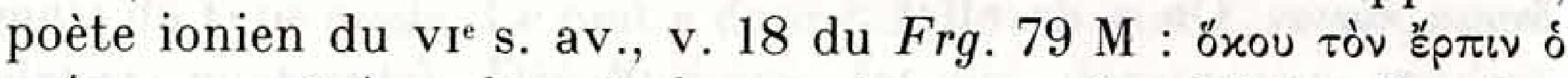

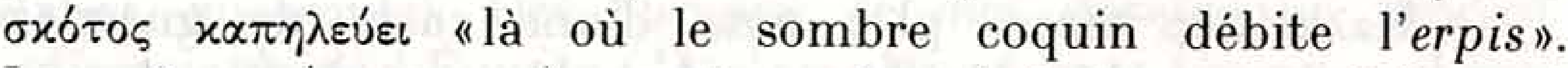
Lycophron (III s. av.), amateur de "glottai», reprend le mot dans son Alexandra, v. 579. Ce vocable devait qualifier un vin médiocre importé d'Égypte ou d'Asie Mineure. Il vient de l'ég. irp "vin" (Wb I 115, 5-7), copte êrp (SBA); cet emprunt est déjà reconnu par les grammairiens anciens ${ }^{14}$.

10. Cf. D'Arcy Thompson, "On Egyptian fish-names used by Greek writers", $J E A, 14$ (1928), p. 22 sqq. Et plus récemment I. Gamer-Wallert, Fische und Fischkulte im alten Ägypten, Wiesbaden, 1970, p. 41.

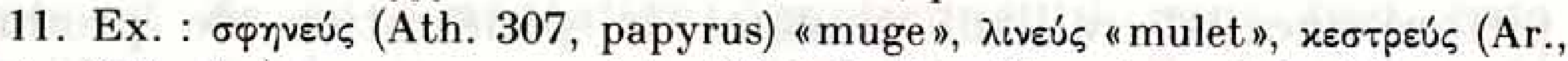
Nuées, 339, etc.) "muge» ou "mulet». Cf. J. L. Perpillou, Les substantifs grecs en - $\varepsilon u_{\varsigma}$, Paris 1973, p. 293 et 321-2. Quelque tentante qu'elle puisse être, nous rejetterons, avec Chantraine, Dict. Étym., l'étymologie évoquée par Perpillou p. 322 :

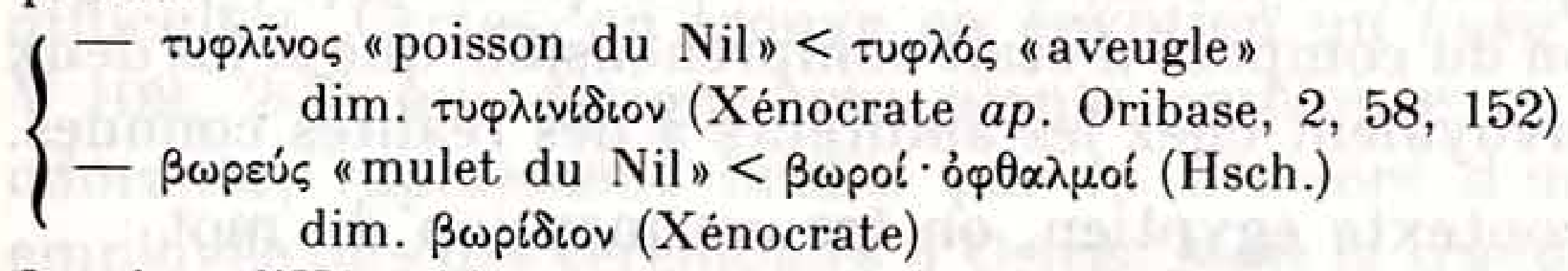

La glose d'Hésychius ne peut être mise en rapport avec $\beta \omega p \varepsilon u ́ s$ : le $\beta$ y note en effet un digamma.

12. L'aspiration que présente l'égyptien disparaît en grec, comme c'est généralement le cas ainsi que l'a montré Sethe, "Zur Wiedergabe des ägyptischen $h$ am Wortanfang durch die Griechen ", Gött. Nachr. 1925, p. 56. Cf. aussi ibıs.

13. Spiegelberg, 1907, p. 131 et Nencioni, p. 11-12. Sur l'espèce d'ébène égyptienne, cf. A. Lucas, Ancient Egyptian Materials and Industries, 1962, p. $434 \mathrm{sq}$.

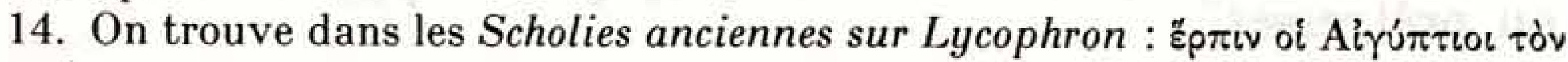
olvov (Lycophronis Alexandra, éd. Kinkel 1880, p. 123). Les Byzantins Jean Tzetzès (Scholies sur Lycophron) et Eustathe ont eux aussi signalé l'origine égyptienne du mot. Ce sont d'ailleurs les grammairiens qui ont, par une analogie plaisante avec le verbe ह̌p $\pi \omega$ "ramper», ajouté une aspiration initiale qui se retrouve dans tous les manuscrits sous la forme žprıs: comme le dit Tzetzès,

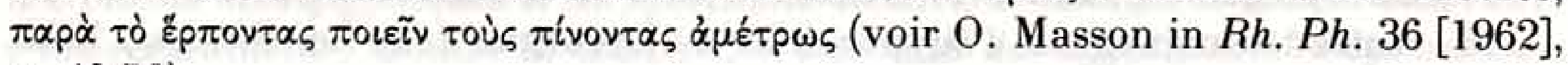
p. $46-50)$. 


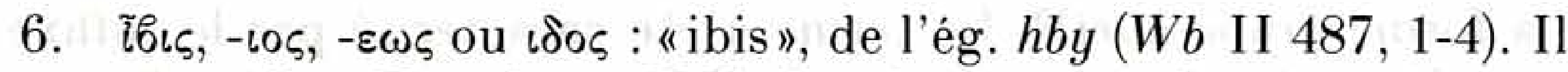
apparaît chez Hérodote, II 75-6 qui n'a cependant pas dû être l'introducteur du mot dans la langue grecque. Si, dans sa description de la faune égyptienne, il cite le nom indigène du crocodile en ménageant l'étrangeté du mot par l'expression

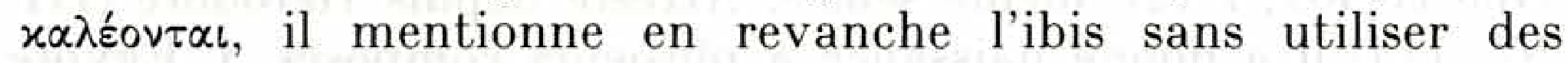
précautions qui laisseraient entendre que le nom était, à son époque, nouveau ou peu connu.

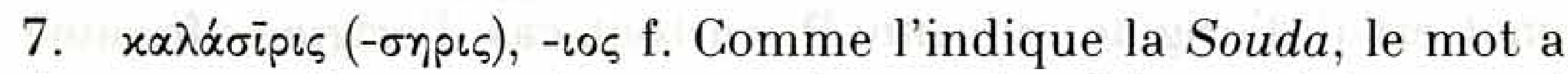

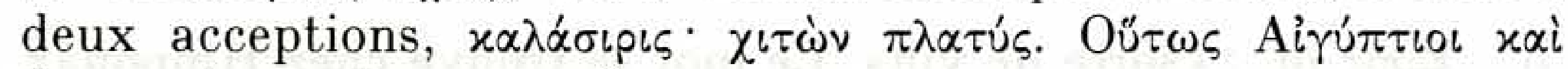
óvo $\mu \alpha$ xúpıov. On a en effet:

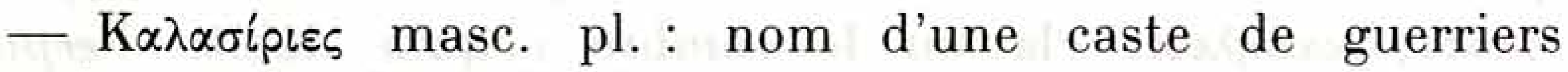
mentionnée par Hdt. II 164, issu probablement de l'ég. kry-šry (Wb V 135, 1), dém. glr-šr "guerrier, soldat" (E 588), copte čalašire "guerrier, géant»"

- $x \alpha \lambda \dot{\alpha} \sigma \iota p ı \varsigma \mathrm{f}$. "vêtement égyptien, large et garni de franges" (Hsch. $\chi \iota \tau \dot{\omega} \nu \pi \lambda \alpha \tau \dot{\sigma} \sigma \eta \mu \circ)$ ). Terme attesté chez Hdt. II 81 ; chez

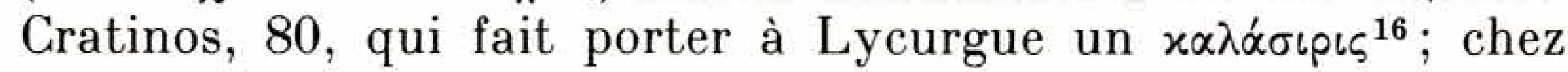
Alexis qui en fait le titre d'une de ses comédies ( $a p$. Pollux, $X$ 18). Le mot devait désigner une tunique égyptienne de grand prix. Son emploi chez les comiques, gourmands de détails pittoresques et raillant souvent la pénétration de produits exotiques dans la vie quotidienne, démontre la relative assimilation du vocable, soulignée par deux autres éléments :

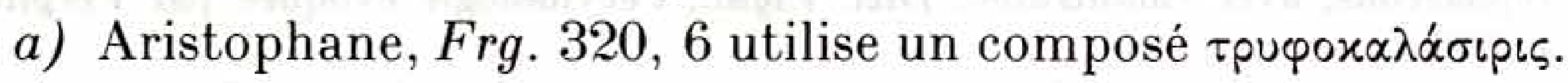
Or la création du composé n'était compréhensible que si les deux éléments renvoyaient chez les auditeurs à des réalités connues.

b) Hors contexte égyptien, on trouve mention du mot:

- Chez Démocrite d'Éphèse, 1 (ap. Athénée, XII 525d) dans son ouvrage Sur le temple d'Éphèse : il a dû finir par désigner un certain type de vêtement de luxe, de travail plus généralement oriental.

- Dans l'inscription des mystères d'Andanie en Messénie,

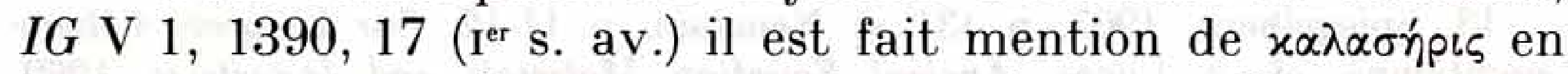
tant que tuniques d'apparat portées par de jeunes filles, esclaves ou prêtresses.

15. Cf. Spiegelberg, $Z A ̈ S, 43$ (1906), p. 86-7 et Wiedemann, p. 24.

16. Autre témoignage de l'égyptophilie de Lycurgue dans Les Oiseaux, 1296, où il est surnommé ïbıs. 
L'étymologie du mot est délicate. Il est certes jugé égyptien depuis l'Antiquité (Hdt. II 82; Sch. Ar. Av. 1294; Pollux, VII 71; Photius; la Souda) mais aucun correspondant n'a été découvert. Peut-être faut-il, avec Chantraine (Dict. étym.), faire dériver le nom du vêtement de celui de la caste de guerriers qui l'auraient porté.

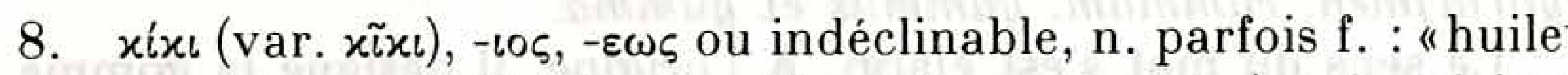
de ricin» (Hdt. II 94 ; Platon, Tim. 60a ; pap.; etc.) puis "ricin" (Str. XVII 2, 5; Diosc. IV 161; etc.) Aétius et Paul d'Égine désignent la plante sous le nom de $x \iota x \varepsilon ́ \alpha f$., par analogie avec les

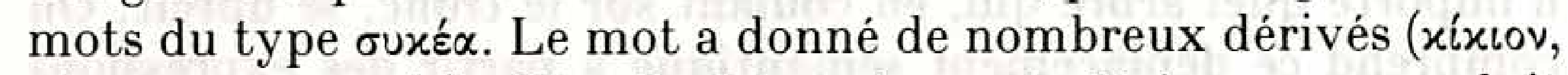
xíxเvos, xเxเouprós). Dès l'origine, le mot désigne un produit

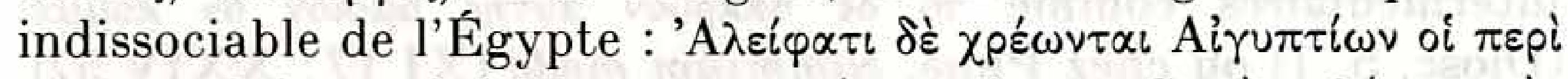

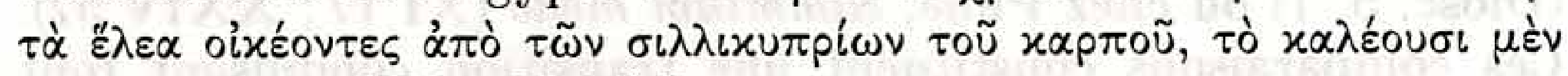

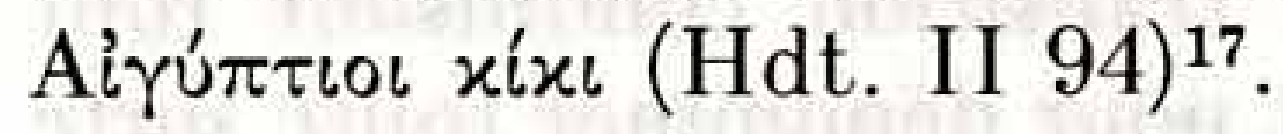

Mais plus tard Platon, Timée, 60a, nous apporte la preuve de l'emploi quasi courant de ce mot. Sans faire allusion à l'Égypte,

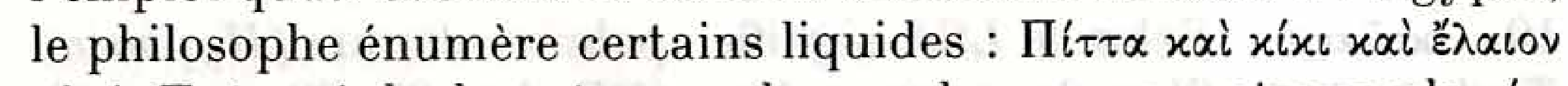
aúcó. Entouré de deux termes de grand usage, on retrouve le xíx qui, puisque non expliqué, devait être parfaitement connu des Athéniens. Pour rendre compte à la fois de son utilisation par les Grecs et de sa connotation égyptienne, il nous faut bien considérer le $x i ́ x \iota$ comme un produit occasionnellement importé d'Égypte.

L'identification du correspondant égyptien offre quelques difficultés. Certes, on trouve en égyptien un kyky ou $k 3 k 3$ (Wb V 109, 2-7) qui désigne une plante employée en pharmacie, odorante, rapidement brûlée par le feu lors d'une tempête, employée lors des cérémonies d'Osiris. Mais la traduction exacte de ce mot n'est pas assurée. D'autre part, le nom égyptien du ricin paraît être dgm (Wb V 500, 9-13), copte tičmes (S). En fait les caractéristiques de la plante kyky correspondent à la matière du ricin. De plus kyky a donné le copte kiki, kuki (B) "ricin». On aurait alors en copte la répartition sémantique suivante : kiki "graine de ricin» et tičmes "ricin", répartition qui reflète peutêtre celle de l'égyptien kyky/dgm neutralisée en $\operatorname{grec}^{18}$.

17. Pour les autres auteurs, cf. R. Dawson in Aegyptus, 10 (1929), 47-72.

18. Cf. Loret, $A S A E, 1901$, p. 11 no 192 et La flore pharaonique d'après les documents hiéroglyphiques et les spécimens découverts dans les tombes, Paris 1892. Plus récemment: Deines et Grapow, Wörterbuch des ägyptischen Drogennamen, Berlin 1959, s.u. 
9. xó $\mu \mu \iota$, le plus souvent indéclinable ou $-\varepsilon \omega \varsigma, n$. : "gomme produite par l'Acacia Nilotica», de l'ég. lımy.t "gomme» (Wb $\mathrm{V} 39,3)$, copte komme $(\mathrm{S})$, komi $(\mathrm{B})$. Le terme a donné matière à

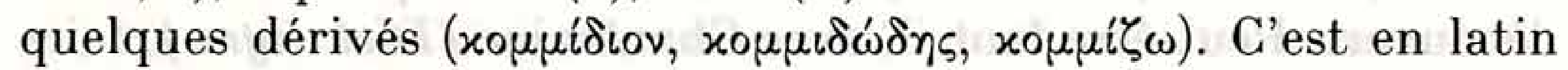
qu'il s'est le mieux assimilé : à côté de cummi, on trouve de bonne heure cummis (Caton, De agricultura, 69), plus tard $g u(m) m e n$, cummum, gummus et gumma.

Le sens du mot s'est élargi. A l'origine, il désigne la gomme produite par l' '’x $\alpha \nu \theta \alpha$ (Hdt., Thphr., Diosc., Str., Ath.) et l' $\alpha x \alpha x i \alpha$ (Diosc. 1, 101). Par extension, il en arrive à désigner la sève de n'importe quel arbre qui, en coulant sur le tronc, se durcit. On comprend ce déplacement sémantique à partir des expressions

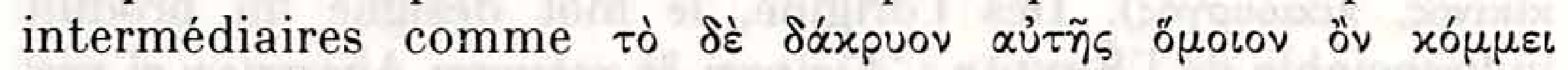
(Diosc. 5, 1) ou chez Pline, cummium modo (XI 17; XXIV 57). Ces comparaisons constituent une transition conduisant tout naturellement à l'utilisation du mot pour dénommer toute sorte de sève mucilagineuse.

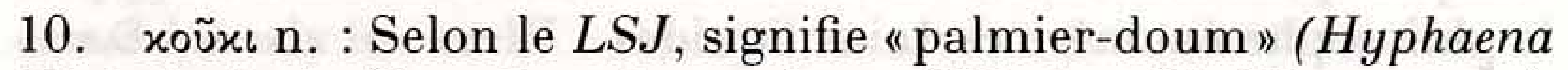
Thebaïca) et "fibre du même arbre» (PBaden, 35, 23, I $^{\text {er }}$ s. ap.). Issu de l'ég. ḳwḳw (Wb V 21, 14-15) "fruit du palmier-doum», dém. $k k$ (E 569), copte kouk, čouč (S) «noix de palmier-doum» ${ }^{\mathbf{1 9}}$.

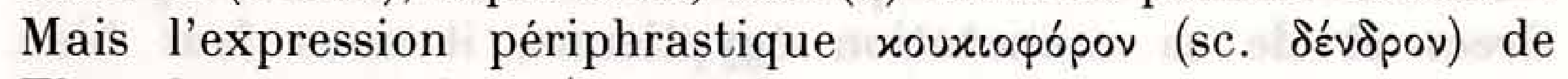
Théophraste, 4, 2, 7 (première occurrence d'un mot de cette famille) prouve que xoũx, contrairement au cuci de Pline, XIII 62, devait signifier "fruit du palmier-doum». Ce sens est

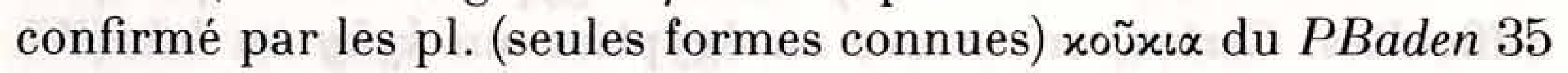

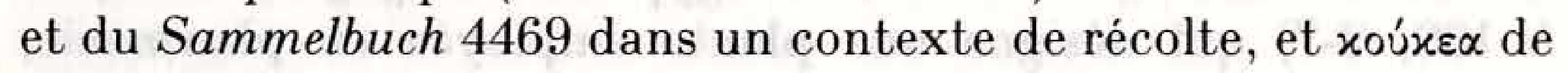
l'Ostr. Wilbour 76 concernant une vente de fruits. Le nom. sing. est déduit de ces formes et garanti par le cuci de Pline.

Le POxy. 1742, $7\left(\mathrm{IV}^{\mathrm{P}}\right)$, le Periplus Maris Rubri, 33 et prob. Strabon XVII 2, 7 donnent le dérivé xoúxเvos.

11. $\chi \cup \lambda \lambda \tilde{\eta} \sigma \tau \iota \varsigma,-\iota \circ \varsigma \mathrm{m}$. : "pain égyptien fait d'ő $\lambda u p \alpha »$, de l'ég. kršt $\left(W b\right.$ V 136, 2-3) «sorte de pain » ${ }^{20}$. Les renseignements que nous donne Athénée en III 114 et X 418 nous permettent d'établir pour notre mot la chronologie suivante: Hécatée (vi ${ }^{\mathrm{e}} \mathrm{s}$. av.) semble le premier à avoir employé le mot (323 (b) Jacoby), le rapportant d'Égypte pour les besoins de ses enquêtes. Puis

19. Cf. Ingrid Wallert, Die Palmen in alten Ägypten, Berlin 1962, p. 52 et N. Hohlwein, "Palmiers et palmeraies», Et. Pap. 5 (1939) 22-7.

20. Cette filiation est déjà décelée par les grammairiens grecs : Pollux, VI 73, Hsch. s.u., Ét. Magn., s.u. ह่ $\pi \iota x u ́ \lambda \lambda \omega \mu \alpha$. 
Hérodote, imprégné de l'ouvrage d'Hécatée, le reprend, faisant entrer le $x \cup \lambda \lambda \tilde{n} \sigma \tau \iota \zeta$ dans la sphère des connaissances qui est celle d'un Grec, en particulier d'un Athénien : Aristophane en effet le cite dans les Danaïdes et l'historien Phanodémos (IV ${ }^{\text {e }}$ s. av.) en fait mention dans son Histoire de l'Attique. Le poète épique Nicandre (II ${ }^{e}$ s. av.) l'utilise lui aussi. Enfin, les papyrus nous livrent plusieurs fois le terme, ce qui s'explique par le fait que le mot était déjà introduit en grec et qu'il correspondait à une réalité à laquelle les Grecs d'Égypte devaient faire référence ${ }^{21}$.

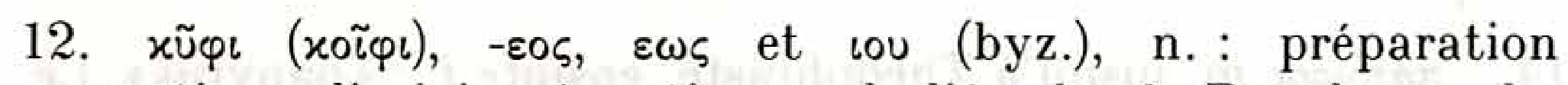
aromatique d'origine égyptienne, de l'ég. $k 3 p . t$ "Rauchenwerk" et "Rauchermittel in der Medizin" (Wb V 104, 1-2). Il s'agit d'une composition ressemblant à de l'encens que l'on faisait brûler dans des cérémonies religieuses et qui s'utilisa aussi comme préparation médicale sous forme de fumigation. Le premier auteur de langue grecque à en faire mention est le prêtre égyptien Manéthon (III ${ }^{\mathrm{e}} \mathrm{s}$. av.). Cf. la Souda, s.u.

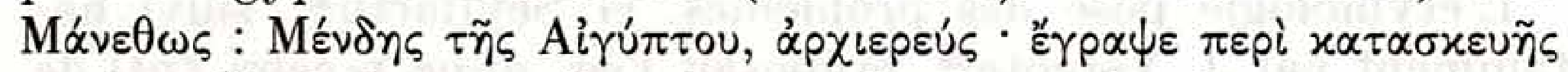

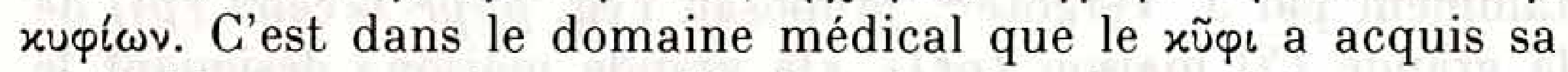
véritable renommée et qu'il s'est implanté en Grèce comme thérapeutique courante: Dioscoride, 1, 25; Damocratès ap. Galien, 14, 117; Galien, 13, 19; Alexandre de Tralles. Il est vraisemblable que le $x \tilde{\varphi} \varphi$ est passé dans la civilisation grecque par le canal de la religion et celui de la médecine, intimement liés. Le témoignage d'Aelius Aristide est à cet égard révélateur : lors d'un séjour à l'Asklépéion de Smyrne, on lui prescrit du

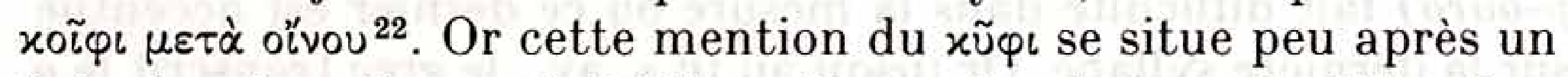
épisode "égyptien": Aristide voit en rêve le temple d'Apollon sur le mont Milyas. Mais au lieu du prêtre du dieu grec, il voit celui d'Isis à Smyrne. De plus Asklépios a été très vite asimilé à Sarapis, les deux dieux entrant souvent en rapport syncrétique. Cet exemple riche en coïncidences nous engage à formuler l'hypothèse selon laquelle l'introduction du xũ rendue possible par les relations privilégiées qui unirent le culte d'Asklépios et ceux de divinités égyptiennes, ainsi que par l'engouement des médecins, en contact direct ou indirect avec la civilisation égyptienne.

21. $U P Z 46,15 ; 53,15$ ( $\mathrm{II}^{\mathrm{e}} \mathrm{s}$. av.) probablement aussi dans le P.Oxy. 1742, 1 (II s. av.).

22. I ${ }^{e r}$ discours sacré, XLVII, § 26, p. 211 (éd. Behr). 
Son assimilation en grec est parfaite : hellénisé dans sa flexion, il a donné lieu à un composé xuழosıońs et au dérivé xupóviov (Alexandre de Tralles, De Chron. I 10). Au sujet des différences orthographiques, on lit dans l'Etymologicum $M a$ -

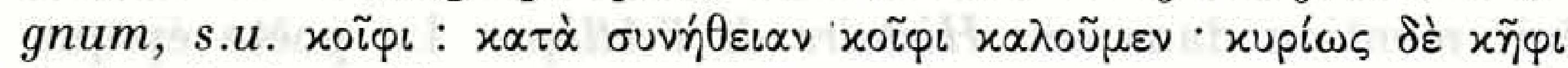

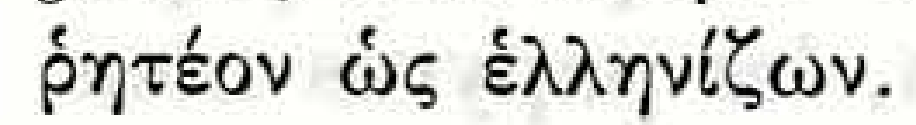

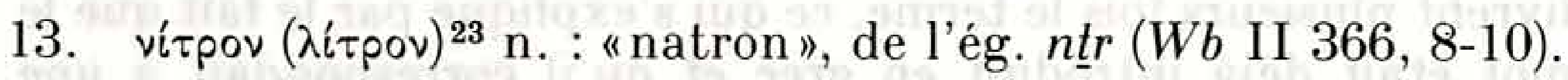
Le terme est attesté déjà chez Sappho et a produit de nombreux composés ${ }^{24}$.

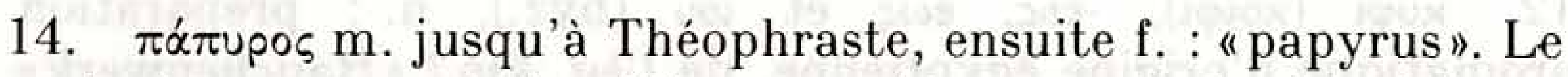
mot $\pi \alpha \dot{\alpha} \pi$ upos a peut-être été emprunté par un botaniste tel que Théophraste (première occurrence dans son Historia Plantarum, $4,8,2)$ pour nommer la plante que l'ancien nom $\beta u ́ b \lambda o s$, désignant aussi le papier fabriqué à partir de l'ombellifère, n'arrivait plus à dénommer dans son acception strictement botanique.

L'étymologie pose des problèmes. G. Seyffarth ${ }^{25}$, suivi notamment par J. Vergote ${ }^{26}$, proposait l'ég. $p 3-p r-\{$ «celui ( $p 3$ ) de la grande (३) maison ( $p r)$ », "la grande maison» désignant le palais royal, puis le roi lui-même. La plante tirerait son nom du fait qu'elle était l'objet d'un monopole royal ou qu'elle était fabriquée dans des manufactures sous contrôle d'État.

Mais J. Vergote en est venu plus tard à modifier son point de vue pour des raisons phonétiques dans "L'étymologie du mot papyrus» ${ }^{27}$. La transcription vocalique grecque de $p \mathbf{3}-p r-\mathfrak{3}\left({ }^{*} p a-\right.$ p-ouro) fait difficulté dans la mesure où ce dernier est accentué sur la dernière syllabe. Or jusqu'au $\mathbf{I I}^{\mathrm{e}} \mathrm{s}$. av., le grec transcrit le $o$

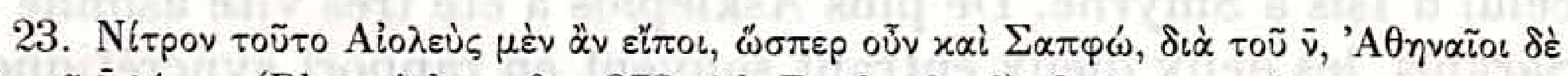
$\delta i \dot{\alpha} \tau o \tilde{~} \bar{\lambda} \lambda i \tau$ pov (Phrynichos, frg. 273, èd. Rutherford). Cette transformation de y en $\lambda$ a été diversement interprétée : 1) Kühner-Blass, I, p. 146, en font un simple changement de $\vee$ en $\lambda$. Ex. : N $\alpha \pi \eta / \Lambda \alpha \pi \eta$ (Hellanicos ap. Hérodien, I 338). 2) Schwyzer, Gr.Gr. I 259 (suivi par Chantraine) préfère y voir une dissimilation de $\nu, \tau$ en $\lambda, \tau$, phénomène qu'il illustre par le seul exemple de vítpov. 3) On peut

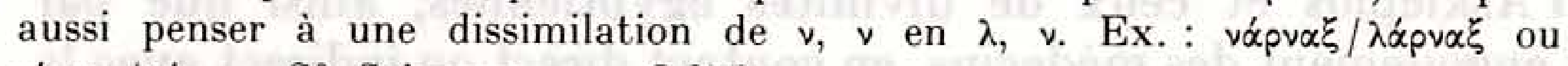
víxvov/ $\lambda$ íxvov. Cf. Schwyzer, o.c. I 259.

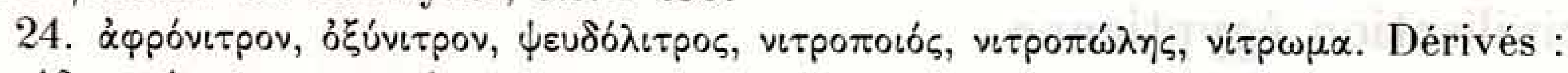

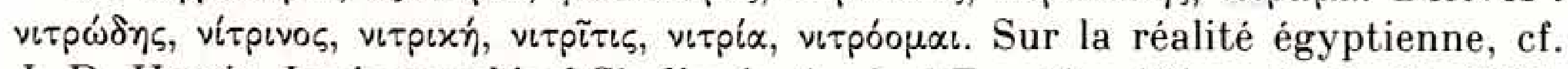
J. R. Harris, Lexicographical Studies in Ancient Egyptian Minerals, Berlin 1961, p. $190-6$.

25. "Ueber das Papier der Alten nach Plinius und der Papyrusstaude im botanischen Garten zu Leipzig" in Serapeum, 3 (1842), p. 33-37, 49-56.

26. "L'origine du mot papier» in Mélanges Grégoire, II (1951), p. 411-6.

27. Chronique d'Égypte, 60 (1985), p. 393-7. 
bref ouvert en syllabe accentuée par $\omega$. D'autre part, la sonante et le $|u|$ atones se transcrivent par le grec $\circ(=o$ fermé $)$. Ainsi, $p$-pr- $\{$ devrait aboutir à $* \pi \alpha \pi \circ \rho \omega s$. Vergote cherche alors le prototype égyptien dans le mot $p 3-p r$, vocalisé ${ }^{*} p a-p \bar{o} r$, «celui de la maison", une autre manière d'exprimer les connexions avec le monopole d'État de la culture du jonc. Le ō fermé doit être normalement rendu par le grec $u$ dans les transcriptions antérieures $\mathrm{au} \mathrm{II}^{\mathrm{e}} \mathrm{s}$. av., car le $v$ est la voyelle arrondie la plus fermée dont disposait alors l'ionien-attique. Ainsi aboutit-on à * $\pi \alpha \pi \tilde{p} \rho \sigma_{\varsigma}$ qui, en vertu de la loi de Vendryes, devient $\pi \alpha \dot{\pi} \pi$ upos.

15. $\sigma \tau i \mu \mu \iota$ ou $\sigma \tau \tilde{\mu} \mu \iota,-\iota \circ \varsigma,-\varepsilon \omega \varsigma$ ou $-\iota \delta \circ \varsigma \mathrm{n}$.; on trouve aussi $\sigma \tau i \mu \mu \iota \varsigma$ ou $\sigma \tau i \tilde{\mu}$ с f. et une fois $\sigma \tau \iota \mu i \alpha$ f. Cyran. 64 ; la Septante emploie toujours $\sigma \tau i b_{l}:$ :poudre d'antimoine pour se faire les yeux",

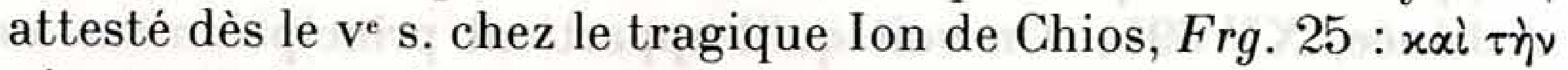

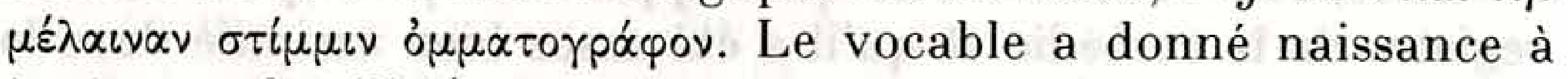
toute une famille $\left(\sigma \tau i \mu \mu \iota \sigma \mu \alpha, \sigma \tau \iota b \iota x \dot{\eta}^{\prime}, \sigma \tau \iota \mu i \zeta \omega\right)$. Il est issu de l'ég. $m s d m . t(W b$ II 153,9$)>s d m^{28}$ "farder les yeux» et "fard» $(W b$ IV $370,3-10)>$ dém. stm (E 478) > copte stèm. La substance désignée par $m s d m . t$ est la galène (en provenance de mines de la mer Rouge).

16. $\sigma \tilde{\omega} \rho \nu$ (var. $\sigma \tilde{\omega} \rho \iota),-\varepsilon \omega \varsigma$ n.: "substance métallique ou métalloïde (probablement du sulfate de fer)". Ce mot est d'abord employé par Dioscoride, 5,10 , puis par Galien et Oribase. Il se retrouve en latin chez Pline et Celse. Pline, XXXIV 120, donne d'utiles renseignements pour identifier plus précisément le sens de $\sigma \tilde{\rho} \rho$ : sory Aegyptium maxime laudatur (...) : quanquam oculorum curationi quidam utilius Cyprium putent. C'est un produit qui dégage une très forte odeur et qui, broyé, devient gras, noir et spongieux. On pense à un ingrédient à usage ophthalmique tel que l'antimoine. Ce dernier se dit en copte basour (B), composé de ba "métal» (< ég. by3) et sour remontant sans aucun doute à l'ég. $s$-wr, certes de sens incertain, mais en tout cas utilisé dans le Papyrus Ebers pour désigner un produit médical contre les maux d'yeux ${ }^{29}$. Le $\sigma \tilde{\omega} \rho u$ serait donc la transcription de l'ég. $s$-wr.

28. Chute du $m$ initial très fréquente : cf. infra $\lambda \varepsilon \mu \varepsilon \tilde{\sigma} \alpha, \lambda \varepsilon \sigma \tilde{\nu}$ เ५.

29. Ebbel, Papyrus Ebers, p. 133. Le $s$-wr désigne aussi dans Dem. Mag. Pap. III 25 un minéral utilisé contre les maladies de peau. Cf. pour la discussion, Deines et Grapow, Wörterbuch der ägyptischen Drogennamen, p. 420-1, ainsi que Harris, Lexicographical Studies in Ancient Egyptian Minerals, p. 179-80. 
Remarquons enfin que le $\sigma \tilde{\omega} \rho u$ appartient au même registre sémantique que le $\sigma \tau i ́ \mu \mu$. On aurait là une production spécialisée typiquement égyptienne.

17. $\psi \alpha \dot{\alpha} \gamma \delta \bar{\alpha} \nu,-\alpha v o \varsigma$ (var. $\psi \alpha \dot{\alpha} \gamma \delta \alpha \varsigma, \sigma \alpha \dot{\alpha} \gamma \delta \alpha \varsigma)$ m. : "sorte d'onguent" d'origine égyptienne, de l'ég. sgnn "onguent" (Wb IV 322, 17),

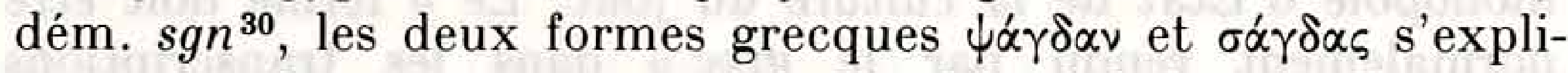
quent par la présence ou non de l'article défini masculin $p(3)$ : on a d'un côté $p(3)$-sgnn $\left(={ }^{*}\right.$ psagn $\left.n\right)$ et de l'autre sgnn $(=$ $\left.*^{*} \operatorname{sagn}^{\circ} n\right)$, les deux, sous l'effet d'une dissimilation, devenant ${ }^{*}$ psagd ${ }^{\circ} n$ et ${ }^{*} \operatorname{sagd}^{\circ} n$.

Le terme n'est pas rare dans la littérature grecque : Eupolis, 198; Epilykios, 1; Aristophane, Frg. 206; Euboulos, 102; Hippocrate cité par Érotien; Nicandre et Théodore cités par Athénée, XV 691c. On voit qu'il appartient principalement au registre de la comédie et qu'il a été assez bien assimilé dans la langue grecque : il est toujours décliné, parfois affecté d'une finale hellénisante en $-\alpha \varsigma$.

La forme $\psi \alpha \dot{\alpha} \delta \delta \alpha$ livrée par Hésychius ( $\psi \alpha \delta \delta \delta \alpha \cdot \dot{\eta} x \iota v \alpha \dot{b} \alpha \rho\llcorner\varsigma)$ doit être une variante dialectale avec assimilation.

\section{B. Mots-transcriptions.}

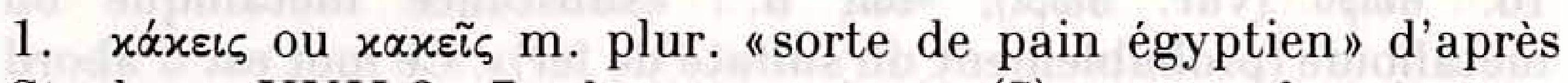
Strabon, XVII 2, 7, du copte čaače (S) "sorte de gâteau", probablement issu du dém. $k^{*} k$ cité par Spiegelberg ${ }^{31}$. Vycichl ${ }^{32}$ propose de remonter à l'ég. " $k k$ "espèce de pain" ( $W b$ I 235,4$)$, lequel provient peut-être de $k$ " $k$ après métathèse.

2. * $\mu \eta \theta i c$ hapax de Plutarque dans De Iside et Osiride, 359B

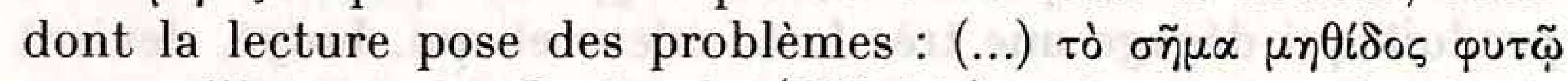

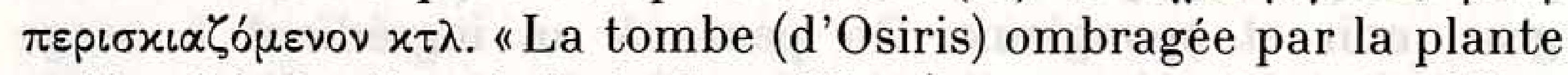
$\mu \eta \theta i \varsigma »$. L'adoption de la lectio $\mu \eta \theta i \delta o \varsigma$ (les manuscrits ont $\mu \eta \theta i \delta \eta \xi$, $\mu \eta \delta(\theta \eta s, \mu \eta \delta เ x \tilde{\eta} s)$ permet de poser un nominatif * $\mu \eta \theta i c$ qu'il est aisé de rapprocher de l'égyptien d'époque grecque $m n t_{3}$ «bosquet sacré de l'abaton d'Osiris sur l'île de Biggeh près de Philae» ( $W b$ II 92, 13-4). Nous avons là une parfaite adéquation entre le texte de Plutarque et les documents égyptiens ${ }^{33}$.

30. Spiegelberg, Hermes, 56 (1921), p. 332-333.

31. Spiegelberg, Cair. Cat. Demot. Pap. 30965 (ptol.) où il traduit le mot par "k$k$-Brote».

32. P. 351 .

33. Cf. Junker, Abaton, p. 51 et 72-3, cité par J. G. Griffith, Plutarch's De Iside et Osiride, Cambridge 1970. De plus, l'absence de nasale en grec est peut- 


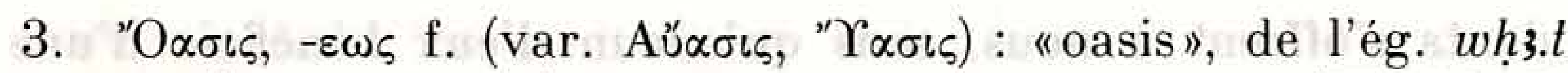
"oasis» (Wb I 347, 18) dérivant de wh3.t "chaudron» (Wb I 347, 12-17). Comparer fr. cuvette comme ustensile et terme géographique $^{34}$. On a le copte ouahe $(\mathrm{S})$, ouehi $(\mathrm{F})$ utilisé comme nom locatif sans article, ce qui n'est pas sans rappeler l'emploi grec sans article ${ }^{35}$. Le terme a toujours conservé en grec une certaine rigidité. Il s'emploie comme un nom propre et a engendré les dérivés 'O $\alpha \sigma i \tau \alpha \iota$, ó $\alpha \sigma \iota \tau \iota x o ́ \varsigma$.

La transcription grecque de $h$ en $\sigma$ est sans précédent. Faut-il y voir l'effort déployé par un Grec pour rendre un son que sa langue ne possédait pas? Le $h$ est en effet une spirante laryngale sourde "différant du $h$ par son caractère confus et rauque qui la fait ressembler au sifflement qui accompagne l'asthme $»^{36}$.

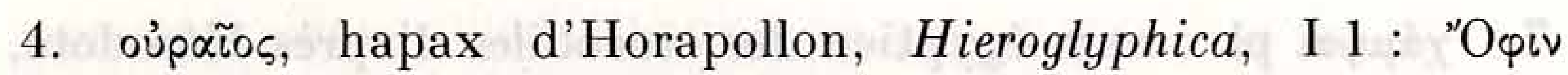

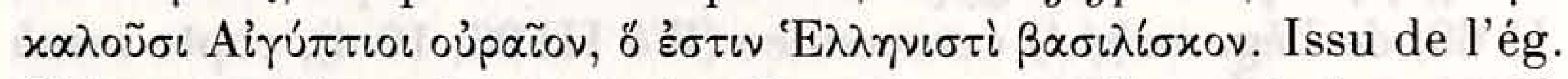
ir.t "serpent" puis par extension "uraeus", à savoir le serpent surmontant la couronne pharaonique ( $W b$ I $42,1-4)$. On peut se demander si Horapollon, en jouant sur le grec, ne met pas le mot oúpaĩos en rapport avec le copte ouro (B) «roi», le traduisant alors par $\beta \alpha \sigma i \lambda i \sigma x о \varsigma$ qui, s'il signifie "serpent», "basilic», n'en vient pas moins de $\beta \alpha \sigma \iota \lambda \varepsilon \varepsilon^{\prime} \varsigma^{37}$.

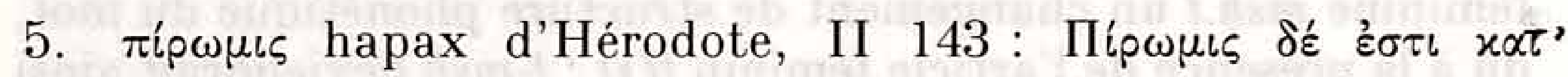

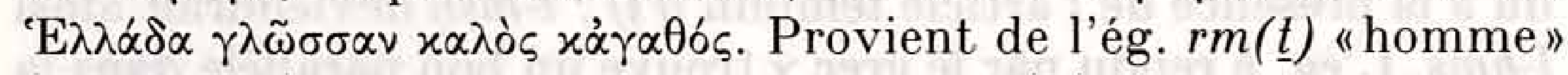
(Wb II 421) survivant dans le copte rōmi (B) précédé de l'article ég. $p_{3}>$ copte $p i-(\mathrm{B})$. L'expression est ici à prendre dans son sens emphatique.

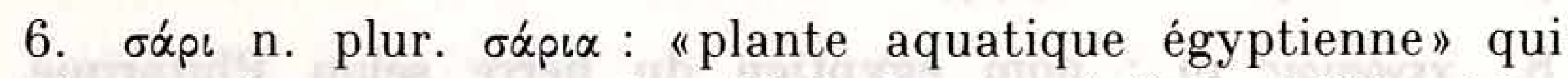
n'apparaît en grec que chez Théophraste, $4,8,5$ et Hésychius (s.u. $\sigma \alpha \dot{\alpha} \rho, \sigma \alpha \dot{\alpha} \rho v)$. Le mot est passé en latin sous la forme sari (var. saripha) employée par Pline en XIII 128. Plusieurs correspon-

être due à un phénomène identique à l'introduction d'un $n$ entre $m$ et $t$ dans certains mots coptes : $m n t$ - à côté de met- où le groupe -nt-représenterait un $/ d /$ (?). Cf. Vergote, Phonétique, p. 103.

34. Cette dérivation est cependant mise en doute par L. Giddy, Suppl. $B I F A O, 1981$, p. 19-28. Cf. aussi plus généralement, Wagner, Les oasis aux époques grecque, romaine et byzantine d'après les documents grecs, 1987, p. 121-4.

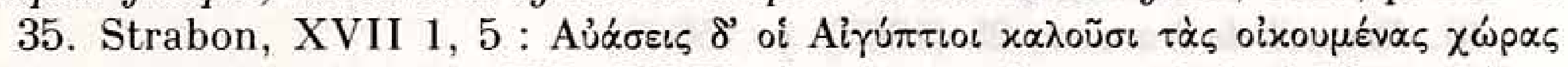

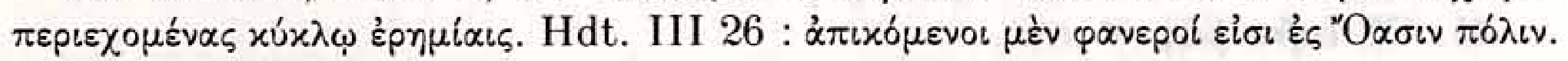

36. Vergote, Phonétique, p. 72 au sujet du $h \bar{a}^{\circ}$ arabe, semblable au $h$ égyptien.

37. C'est l'opinion de B. de Walle et J. Vergote in "Traduction des Hieroglyphica d'Horapollon", Chronique d'Égypte, 18 (1943), p: 41. lontre cite etymologie, J. Leclant, Melanges Maspeno I/4 (1961) p. 76.77 
dants s'offrent à nous, sans qu'aucun d'eux bénéficie d'une traduction précise. A côté de l'ég. $s$ rry "plante médicinale» (Wb IV 19, 10-13), correspondant bien tentant, on trouve aussi $s i r$ "papyrusartige Pflanze» $(W b$ IV 48,4$)$ qui semble désigner le Cyperus Fastigatus en qui l'on doit probablement voir le ó́pı de Théophraste ${ }^{38}$. On a en copte, outre saris "sorte de chicorée», šari que Crum (584a) met en rapport avec le grec et qui désigne une plante aquatique, plus particulièrement un roseau. La précarité sémantique des noms de plantes en égyptien, dont les caractéristiques ne nous sont que très partiellement indiquées par les textes, embrouille la situation. Pour notre part, nous inclinons à penser que le grec trouve dans l'ég. śr un correspondant plus que satisfaisant.

7. $\chi \alpha ́ \alpha \psi \alpha_{\iota}$ pl. : nom égyptien des crocodiles d'après Hérodote, II 69, de l'ég. $m s h(. t)$ "crocodile» (Wb III 96, 10-4), copte msah (S B). Selon Černý ${ }^{39}$ le $\chi \alpha$ - du grec serait l'article indéfini pluriel copte hen- (S), han- (B). Mais ég. $h=\chi$ est une équation sans précédent. Une variante égyptienne avec métathèse $h m s(. t)$, de même sens ( $W b$ III 96, 11-2), paraît bien rendre compte de la forme grecque. P. Lacau ${ }^{40}$, s'il n'y voit qu'une métathèse apparente, de l'ordre du graphisme, accepte pour la forme féminine msh.t un changement de structure phonétique du mot, dû à la présence de l'article féminin $t(3): t$-msh deviendrait ainsi $t$-ḥms. L'ég. $h$ rendu par le grec $\chi$ trouve un bon parallèle dans la transcription que fait le même Hérodote de l'ég. smḥ par $\alpha$ $\sigma \mu \alpha \chi$ (II 20). Enfin le passage du groupe $-m s$ - à $-\mu \psi$ - est aussi illustré par l'exemple de 'P $\alpha \mu \psi \eta \emptyset s<R-m s-s w^{41}$.

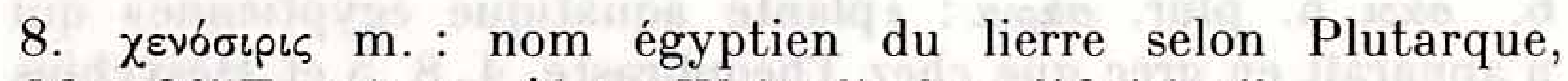
Mor. 365E, soit ég. *h.t-n-Wsir «l'arbre d'Osiris» ${ }^{42}$.

\section{Emprunts du grec d'Égypte et de la Septante.}

1. $\alpha \chi \prec$ n. : "jonc, pré marécageux», attesté dans la Septante (Gen. 41, 2, etc.) et dans les papyrus (P.G.M. IV 1091, 1101),

38. C'est l'avis de Charpentier, Recueil des matériaux épigraphiques relatifs à la botanique de l'Égypte ancienne, Paris 1981, face à Nencioni, p. 9.

39. ASAE, 42 (1943), p. 346-8.

40. Recueil de travaux, 25 (1903), p. 156-9.

41. Cf. infra $\rho \dot{\omega} \psi<r m s$.

42. Newberry, JEA, 15 (1929), p. 93 n. 1, préfère voir dans le premier membre l'ég. ḩ3 qui désigne une plante. Il est suivi par Chantraine, Dict. étym. Mais en fait ḩ3 dénomme la feuille de lotus ou de concombre (Wb III 218, 1-2). 
venant de l'ég. 3hy, 3hy "plante, végétation» $(W b$ I 18, 8) dém. 3hy "fourré de joncs» (E 10), copte ahi, akhi (B), "pré marécageux, joncs». Le mot grec traduit dans la Septante l'hébreu z ăḥu "joncs», lui-même emprunté à l'égyptien ${ }^{43}$.

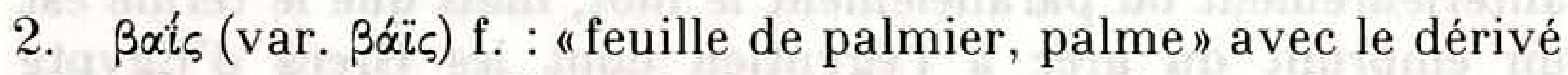
ßáiov de même sens. Il a pris dans les papyrus le sens de "mesure pour arpenter ${ }^{\mathbf{4 4}}$. Présent chez Chaeremon cité par Porphyre, de Abstinentia, IV 7 et Horapollon I 3. Issu de l'ég. $b y$ y, $b i-y(. t)$ (Wb I 446, 9-10) > copte bai (B) «branche de palmier (dépouillée de ses feuilles)" (Crum 27b).

3. ¿iv (var. iv, ziv, giv) n. : nom de mesure égyptienne. On trouve aussi '̌voov (ivıov, ìvóv) que le $L S J$ interprète comme un diminutif, mais qu'il est préférable d'expliquer comme une forme grécisée. "Ivıov se lit dans un fragment de Cléopatre $\left(\mathrm{I}^{\mathrm{p}}\right)$ transmis par le corpus galénique. Provient de l'ég. hnw "pot" qui prend, au Moyen-Empire, le sens d'une mesure de 0,456 l ( $W b$ II 493, 2-14), copte hin (S, B) "récipient, mesure de liquide».

4. xaíuıv n. nom de volaille POxy. $1656,14\left(\mathrm{IV} / \mathrm{V}^{\mathrm{p}}\right)$, du copte ćaime (S) issu probablement de l'ég. gm.t "ibis noir" (Plegadis Falcinellus) (Wb V 166, 5). L'onomastique connaît un nom

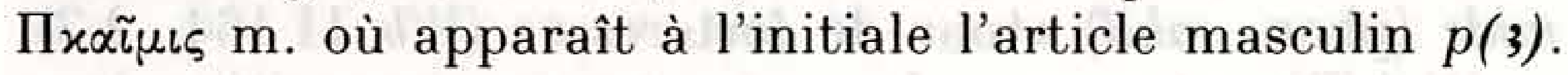

5. $x \lambda \alpha \dot{\lambda}$ เov "collier»: POxy. 114, 11; 796; 1272, 9, PRyl. 154, 7 $\left(\mathrm{I} / \mathrm{II}^{\mathrm{P}}\right)$, etc., issu de dém. ḳll "chaîne» (E. 547, 1), copte klal (Crum 103a). La variante grecque $x \lambda \alpha$ 'vıov est probablement une forme avec dissimilation.

6. xoux(x)oú $\alpha \varsigma,-\alpha \tau \circ \varsigma$ m. : "nom de la huppe en égyptien"

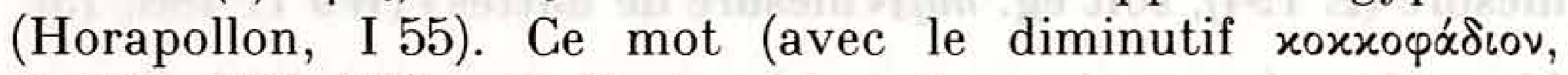
P.G.M. VII, 411) appartient au domaine des papyrus magiques. On trouve le copte koukouphat (B) "huppe» qu'on a cru être formé à partir du grec. Il est vrai que le grec connait, de longue

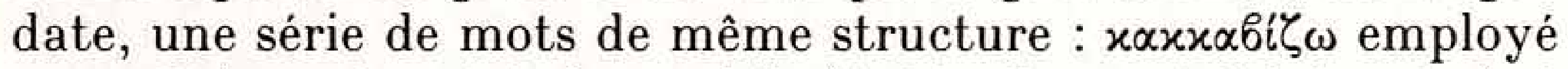
pour désigner le cri de la perdrix (Aristophane, Aristote, etc.) et

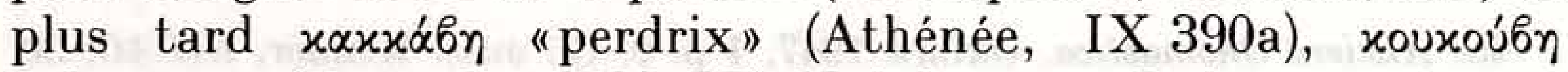
"chouette» (Eustathe, 1523, 59). Mais le copte dérive assurément du dém. k.kpt (E 551) et k̂wḳpt (E 533) «huppe», peut-être issu de l'ég. $k \underline{k}$ nom d'oiseau ( $W b \mathrm{~V}$ 71, 11) que Gardiner traduit par

43. Cf. Vergote, Joseph en Égypte, Louvain 1959, p. 59-66.

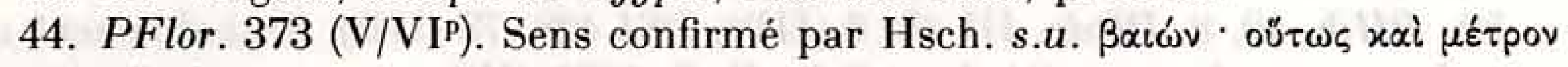
$\pi \alpha \rho$ ' 'А $\lambda \varepsilon \xi \alpha \nu \delta \rho \varepsilon \tilde{\sigma} \sigma$. 
"huppe» attribuant à la deuxième partie -pt une valeur onomatopéique ${ }^{45}$. L'origine égyptienne du mot est démontrée

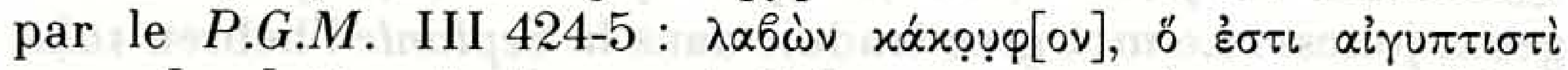
$x \alpha x \times 0 v[\varphi \alpha \tau]$. Il ne faut pas voir là la preuve que le grec possédait antérieurement ou parallèlement le mot, mais que le terme est un emprunt du grec à l'égyptien dont les Grecs d'Égypte auraient perdu conscience.

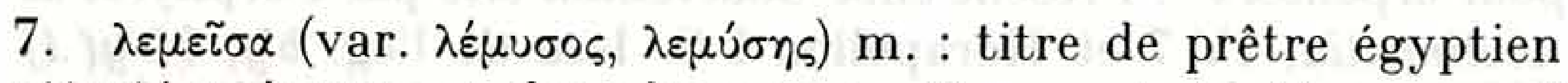
attesté uniquement dans les papyrus ${ }^{46}$ venant de dém. $m r m s^{\text {é }}$ titre de prêtre et titre militaire (E 166), sens qu'il hérite de l'ég. imi-r3 mše "général» (Wb II 94, 5-13); copte lemēše, lemēse "capitaine, héros".

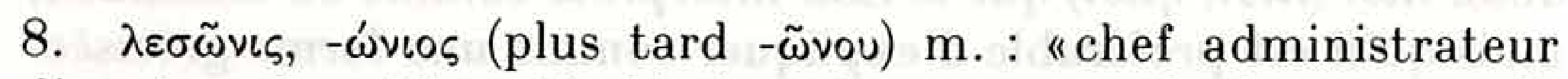
d'un temple», titre attesté dans les papyrus dès le $\mathrm{II}^{\mathrm{e}} \mathrm{s}$. av. ${ }^{47}$. Dérivé : $\lambda \varepsilon \sigma \omega v \varepsilon i \alpha$. Cette création grecque est, dans certains cas,

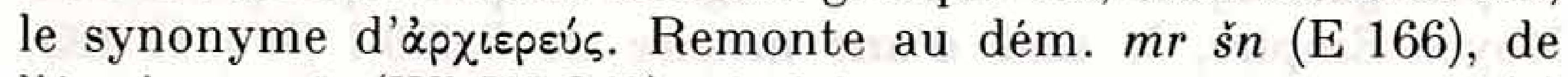
l'ég. imy-r 3 šn (Wb VI 249) "chef de temple». Le copte lašane (S) a perdu son sens religieux pour ne plus signifier que "magistrat dans une localité».

9. $\mu \dot{\alpha} \gamma \delta \omega \lambda$ os $\mathrm{m}$. : "tour de guet» employé dans les papyrus ${ }^{48}$, de l'ég. mktr (phon. mktl) "tour de forteresse» (Wb II 164, 2-3), copte miğtol (B), même sens. Le mot entre en composition dans $\mu \alpha \gamma \delta \omega \lambda \circ \varphi u ́ \lambda \alpha \xi$ et $\pi \cup p \gamma \circ \mu \alpha \gamma \delta \tilde{\omega} \lambda$. Le mot égyptien est lui-même un emprunt à l'hébreu migdāl "tour de forteresse, estrade en bois, armoire, tour de garde d'un vignoble».

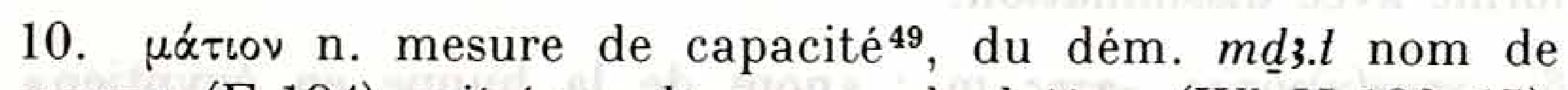
mesure (E 194), soit ég. $m \underline{d} 3$ «mesure de dattes» (Wb II 186, 15).

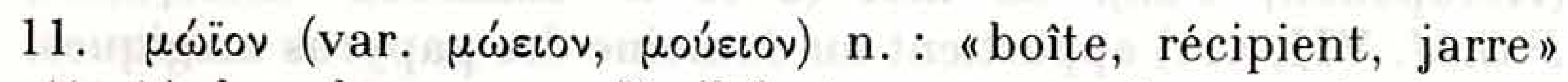
attesté dans les papyrus ${ }^{50}$; il faut en rapprocher aussi $\mu \omega \dot{\sigma} \tau \tau$ iov. Issu de l'ég. $m$ 3̆h nom de mesure (Wb II 31, 1-3), donnant dém.

45. Ancient Onomastica, Oxford 1947, I p. 9. Cf. aussi Keimer, BIFAO, 30 (1931), I p. 318-331.

46. PTeb. 122, $1\left(\mathrm{I}^{\mathrm{a}}\right)$, Sammelb. $1007\left(\mathrm{I}^{\mathrm{P}}\right)$.

47. PAmh. 35, 11; 40, 1; 41, $15\left(\mathrm{II}^{\mathrm{a}}\right)$, Sammelb. 6154, $31\left(\mathrm{I}^{\mathrm{a}}\right)$.

48. BGU 1550 (ptol.) PTheb. Bank I 1, $16\left(\mathrm{II}^{\mathrm{a}}\right)$, Meyer Ostr. 40 (I $\left.\mathrm{I}^{\mathrm{P}}\right)$. PMich.

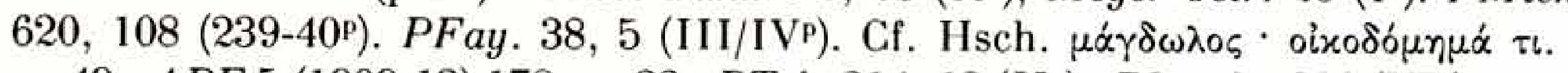

49. APF 5 (1909-13) 178, no 32; PTeb. 314, 18 (II $\left.{ }^{\mathrm{P}}\right)$; PLond. $1906\left(\mathrm{VI}^{\mathrm{P}}\right) ;$ Ostr.

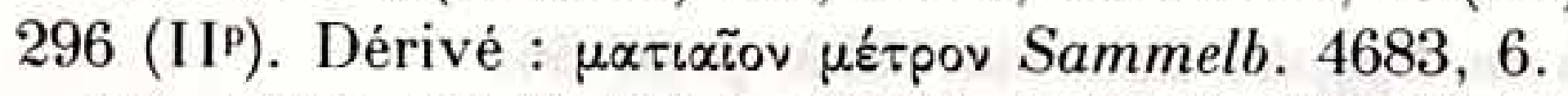

50. PHib. 49, 8, PPetr. III 65, $6\left(\mathrm{III}^{\mathrm{a}}\right)$, PSI 428, $78\left(\mathrm{III}^{\mathrm{a}}\right)$. Probablement au sens de mesure de capacité dans Ostr. Bodl. $1231\left(\mathrm{II}^{\mathrm{a}}\right)$. 
$m y h$ "mesure de fourrage» (E 153), copte moeih, moieiah, nom de mesure. Le sémantisme de récipient n'apparaît pas en égyptien. La mesure déterminée par un contenant a pu engendrer ce nouveau sens.

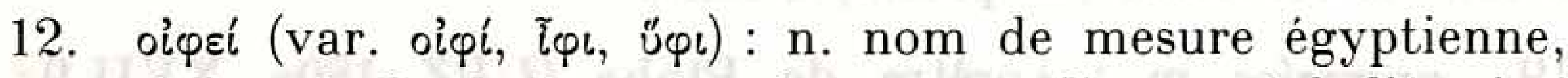
attesté dans la Septante et dans les papyrus ${ }^{51}$ venant de l'ég. ip.t mesure de 40 hin, dém. ipyt (E 29), copte öipi (B) "boisseau».

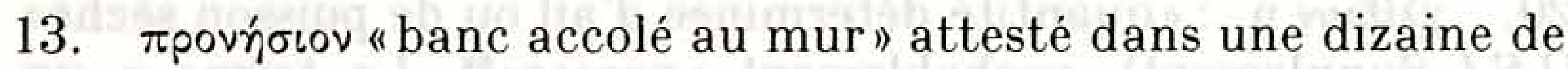
documents (de 11 à 323 ap.) ${ }^{52}$, de l'ég. ns.t "trône, siège" (Wb II 321, 6-323, 15), copte nēse (S) "banc, siège» combiné au préfixe grec $\pi \rho 0^{-}$.

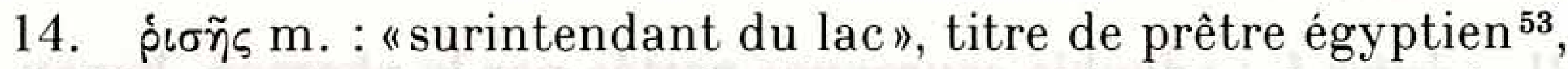

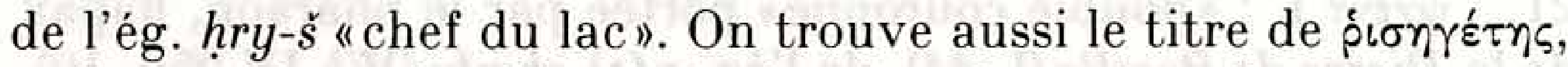

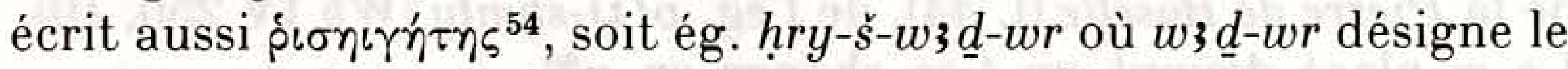
lac Moeris. L'emploi du $\gamma$ pour rendre l'égyptien $w$ est un

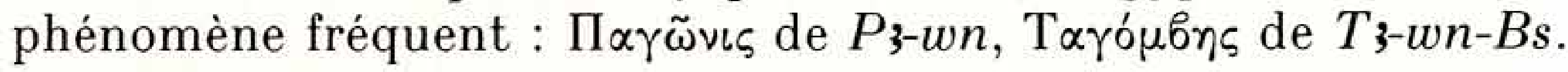

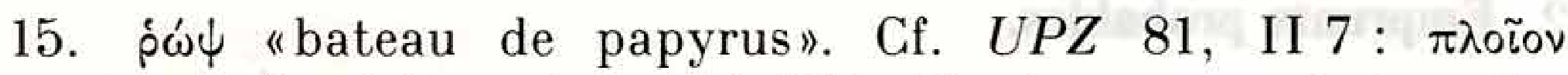
$\pi \alpha \pi \cup ́ p \iota v o v$ oै $x \alpha \lambda \varepsilon i \tau \tau \alpha$ $\alpha i \gamma u \pi \tau \iota \sigma i \hat{\rho} \dot{\omega} \psi$. On trouve aussi dans les

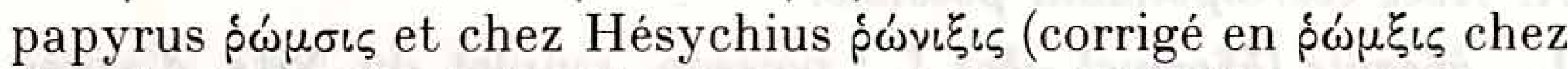
Chantraine). Vient du dém. rms "bateau» (E 242), copte rams.

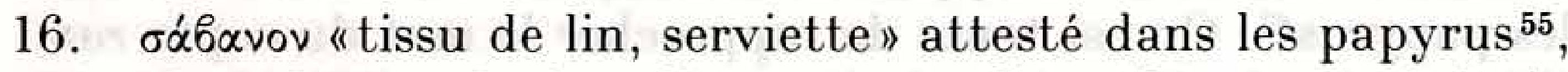
puis chez les médecins d'époque tardive. On a aussi latin sabanum, passé dans le domaine germanique, v. h. all. saban "tissu de lin, linceul». Provient de l'ég. $s b n$ "bandelettes, bandelettes de momie» ( $W b$ IV 89, 13), copte seben (B) «tissu de lin, bande».

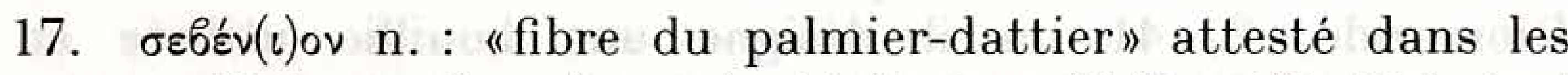
papyrus $^{56}$ et mentionné par Archigène ap. Galien, 12, 574 ainsi

51. Lévitique, 5, 11 ; Ézéchiel, 45, 13; Zacharia, 5, 6; Nombres, 28, 5; Judith,

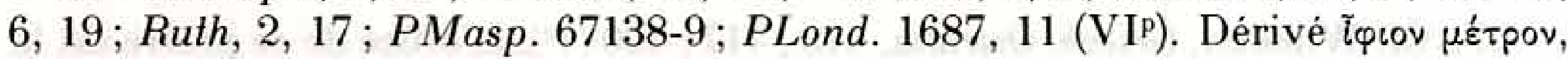
PMasp. 67308, 3 (VI $\left.{ }^{\mathrm{P}}\right)$. Cf. aussi Théognostos, Canones, 18 : oíqvos et Hsch. s.u. oipiv.

52. Cf. G. Husson, Chronique d'Égypte, 51 (1976), p. 167-8.

53. Sammelb. 5231, 2 (IP) et alibi.

54. Sammelb. $i b$. et $5246,3\left(\mathrm{III} / \mathrm{II}^{\mathrm{a}}\right)$.

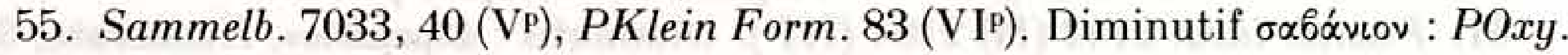

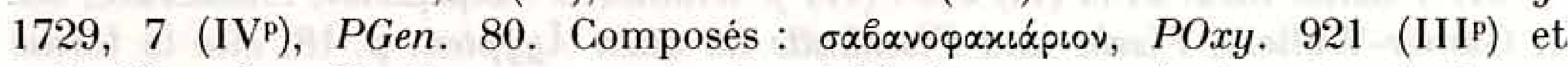

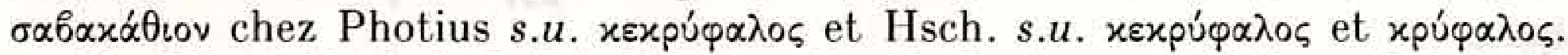

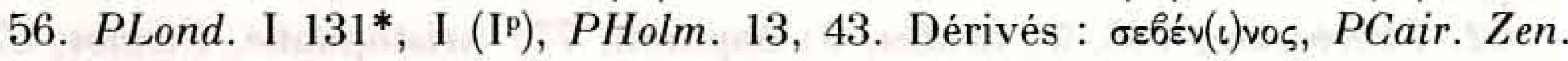
$59438\left(\mathrm{III}^{\mathrm{a}}\right)$. 
que par Hsch. s.u., soit l'ég. šny-bnry (Wb IV 500, 15), copte šenbeni (B) de même sens.

18. $\varphi \varepsilon v \vee \tilde{\eta} \sigma \iota \varsigma(-\tilde{\eta} \sigma \iota,-\hat{\eta} \sigma \iota \varsigma)$ m. : "prêtre d'Isis» ${ }^{57}$, de l'ég. $p(3)-h m$ $n$-Is.t «le serviteur (= prêtre) d'Isis».

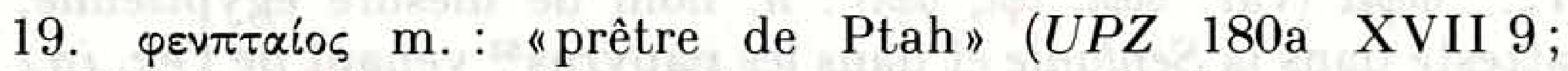
XXX 1), de l'ég. p(3)-ḥm-Pth "serviteur de Ptah".

20. $\chi \lambda$ íbı $\mathrm{n}$. : "quantité déterminée d'ail ou de poisson séché» (LSJ Supplement), probablement "panier" ${ }^{58}$. Le terme a un rapport avec le copte čleb $(\mathrm{F})$ "muselière", sens annexe - ou peut-être dérivé - à celui, perdu, de corbeille (cf. all. Maulkorb) ${ }^{59}$.

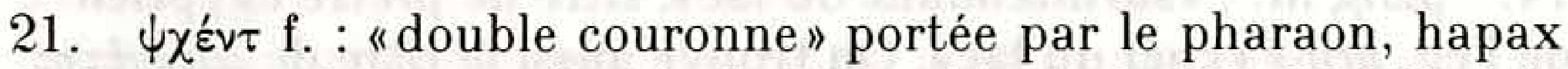
de la Pierre de Rosette (1. 44), de l'ég. p(3)-shmty (Wb IV 250, 10). Le $m$ placé devant un $t$ se change en $n^{60}$.

\section{Emprunts probables.}

\section{A. Avec correspondant possible.}

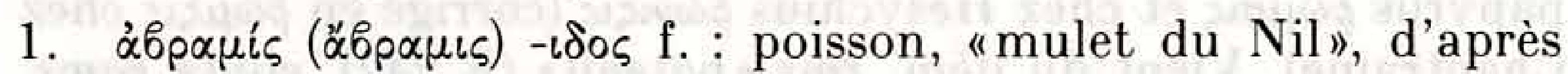
Athénée, VII 312b et Oppien, Halieutiques, I 244; attesté dans les papyrus ${ }^{61}$. On est tenté de rapprocher le mot du copte rami (B) "perche du Nil», issu de l'ég. $r m$ "poisson" (Wb II 416, 1217), accompagné de l'article masculin $p(3)$. On s'explique moins bien la présence d'un $\alpha$ initial, tout comme pour le mot suivant.

2. $\dot{\alpha} \theta \dot{\alpha} \rho \eta$ ou $\alpha \theta n \hat{n} \rho \alpha$ f. : "bouillie de farine ou de gruau», attesté dès le $\mathrm{v}^{\mathrm{e}} \mathrm{s}$. chez les Comiques ${ }^{62}$. Le terme se retrouve chez Dioscoride, 2, 41 où il désigne une bouillie utilisée en cataplasme. Pline, XXII 121, en fait un medicamentum. La Souda (s.u. $\mu \varepsilon \mu \nu \sigma \tau \iota \lambda \eta \mu \varepsilon ́ v o \iota)$ nous dit que l'annp était consommée

57. Cf. Wilcken, Griech. Ostraka, $413 ; 417 ; 420\left(\mathrm{I}^{\mathrm{p}}\right)$. L'office de ce prêtre est la

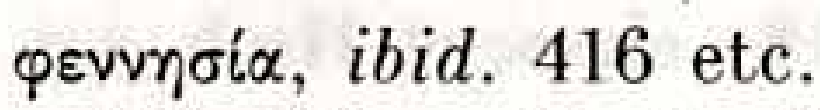

58. PFay. 72, 4; PTeb. 413, 14; POxy. 936, 6.

59. Cf. Stucker, "Trois études», Acta Orientalia, 15 (1936), p. 1-20 cité par Vychichl, p. 337-8.

60. Cf. Sethe, Verbum. §220 et Vergote, Phonétique, p. 103.

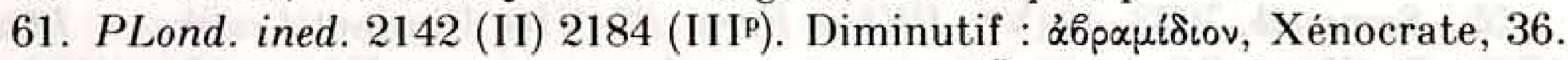
I. Gamer-Wallert, Fische und Fischkulte im alten Ägypten, p. 18, cite la forme ¿’óspuis.

62. Phérécrate, 108, 3; Cratès, 9; Sophron, 77; Aristophane, Ploutos, 673, 683-4; Nicophron, 15; Anaximandre, 4; Hellanicos, frg. 192 Jacoby, etc. 
lors de certaines fêtes; Hésychius s.u., Eustathe, Cassien, Conl. 15, 10, 1 parlent d'une origine égyptienne, confirmée par saint Jérôme d'après lequel $\alpha \dot{\alpha} \hat{p} \rho \alpha$ vient de l'ég. $\theta \eta p$ "farine» ${ }^{63}$. Il existe en effet un tr.t "farine fine et pure» ( $W b$ V 388-7) ainsi qu'un try "sorte de pâtisserie» ( $W b$ V 386,5$)$. De plus le mot grec a des attestations dans les papyrus.

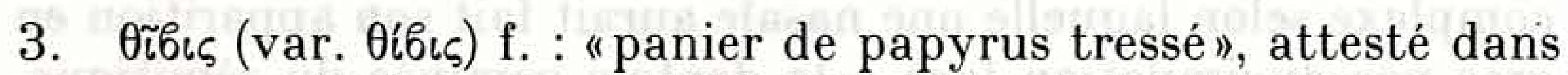
les papyrus (PCair. Zen. $59069 \mathrm{III}^{\mathrm{a}}$, PGrenf. 14, 10, $\mathrm{II}^{\mathrm{p}}$ ) et dans la Septante (qui donne aussi $\theta \dot{b} b \eta$ ). Le mot a été presque unanimement considéré comme emprunté à l'hébreu tēba "caisse, coffre», découlant de l'ég. $\underline{d} b 3 . t$ "caisse» (Wb V 561, 8-9). Mais sa présence dans les papyrus d'Égypte incite à chercher un correspondant égyptien. Or ég. dbs.t et dém. tby.t (E 622) aboutissent au copte thēbi, thebi (B), étonnamment proche du grec.

4. $x \tilde{\eta} b \circ \varsigma$ (var. $x \tilde{\eta} \pi \circ \varsigma) \mathrm{m}$. : "singe à longue queue» (peut-être le Cercopithecus Pyrrhonotus) chez Aristote, HA 502a; Strabon, XVI 4, 16 ; Diodore, III 35, 6 ; Élien, NA XVII 8, etc. Outre le skr. kapīh, l'hébreu $q \bar{o} p(h)$, l'égyptien offre un bon correspondant, avec gyf "petit singe" ( $W b$ V 158 12-3), variantes $g f$, gwf, $g 3 f i$; dém. ǩwf et kwf (E 533 et 562), copte čapi (S). Le vocalisme de l'égyptien invite à préférer l'hypothèse égyptienne. De plus

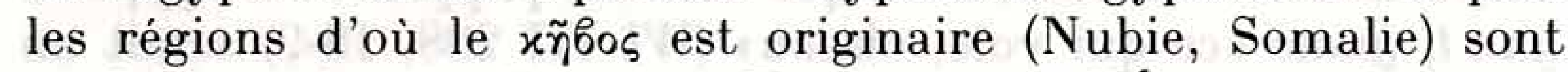
exploitées depuis l'Ancien Empire par l'Égypte. Le nom égyptien est peut-être un emprunt au pays d'où ce singe tire ses origines.

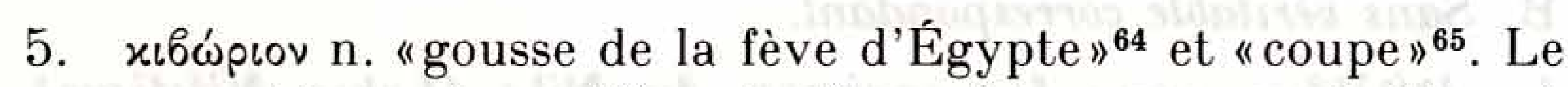

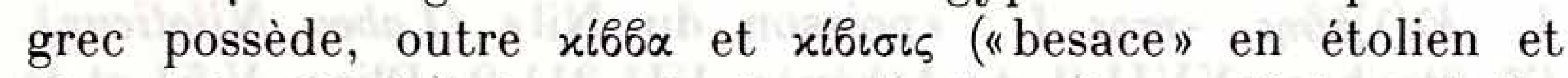
chypriote, Hsch.), des mots de radical analogue désignant des

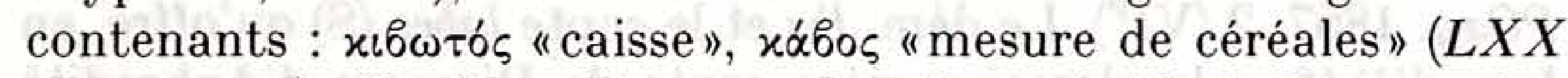
et papyrus). Or l'égyptien possède le mot kiby.t, $k b$ "cruche, mesure pour liquide" (Wb V 25, 2-6), copte kabi, kēbi(B), probablement d'origine sémitique.

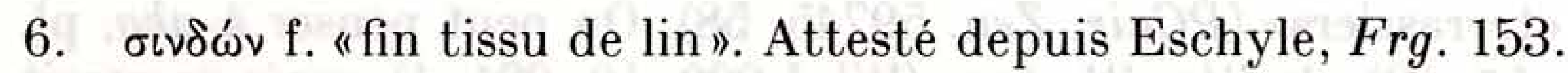
E. Masson ${ }^{66}$ opte pour une étymologie sémitique, évoquant

63. Quaestiones in Genesin, II col. 543 (éd. Martian).

64. Nicandre, frg. 81, 3; Diphilus de Siphnos ap. Athénée, III 73a; POxy. $105,18\left(\mathrm{II}^{\mathrm{p}}\right)$.

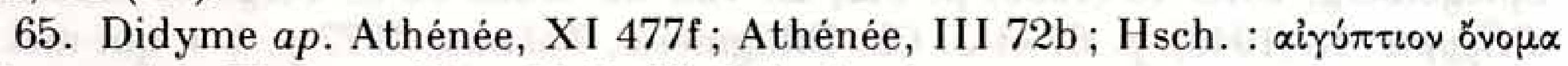

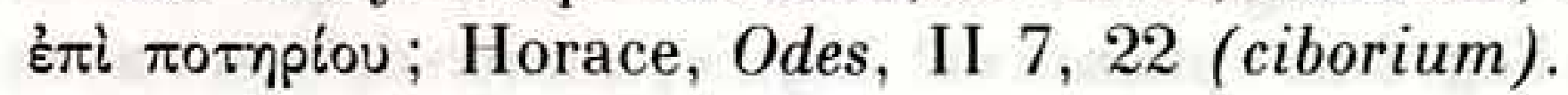

66. Recherches sur les plus anciens emprunts sémitiques en grec, Paris 1967, p. $25-6$. 
l'akk. saddinu/sattinu. L'hypothèse d'un emprunt à l'égyptien šndwt "pagne» (Wb IV 522) nous paraît plus satisfaisante. Le vocalisme du mot, tel que le copte šentō (B) "lin, vêtement» le fournit, épouse beaucoup mieux le grec que les correspondants sémitiques. De plus l'égyptien possède une nasale, absente du sémitique, et permet de faire l'économie de l'explication complexe selon laquelle une nasale aurait fait son apparition en grec par dissimilation face à la dentale géminée du sémitique. Enfin signalons que Pollux, VII 72 fait entrer le $\sigma \iota v \delta \omega \nu$ dans la catégorie des vêtements égyptiens. C'est d'ailleurs par le copte

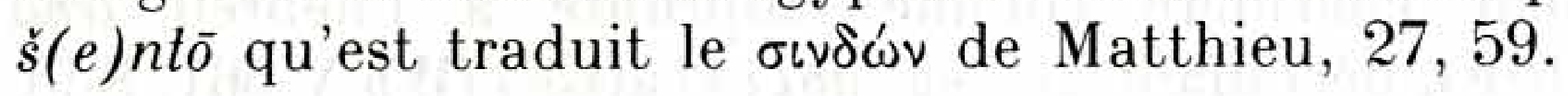

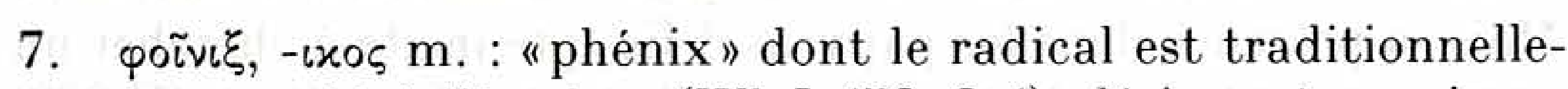
ment rapporté à l'ég. bnw ( $W b$ I 458, 3-4), désignant un oiseau fabuleux qui s'engendre spontanément et ressuscite après la mort. De plus certaines graphies prouvent la présence d'un $y$ avant la nasale, permettant de reconstruire un *boine issu de *boynew, tout comme l'ég. bn.t "harpe", écrit aussi byn.t, a pu donner le copte boine $(\mathrm{S})^{67}$.

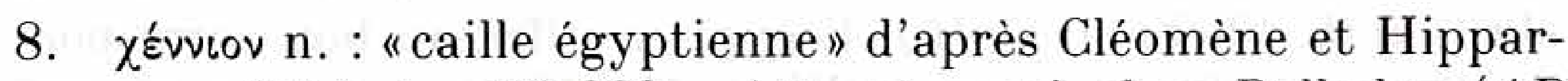
que ap. Athénée, IX 393c. Attesté aussi chez Palladas ( $A P$ IX 377 , v. 7 et 8) et Hsch. s.u., qui en fait un oiseau d'Égypte consommé en saumure, ainsi que dans les papyrus ${ }^{68}$. L'égyptien hnn.t, hnnw "ce qui vole, oiseau" (Wb III 288, 18-9) serait un bon correspondant, quoique le sens égyptien soit trop vague pour avoir désigné une espèce en particulier.

\section{B. Sans véritable correspondant.}

1. $\dot{\alpha} \lambda(\lambda) \alpha \dot{\alpha} \eta_{\zeta},-\eta \tau \circ \varsigma$ f. : "poisson du Nil» (Labeo Niloticus). Cf. Strabon, XVII 2, 4; Athénée, VII 311-2; Pline, V 51 et le POxy. 1857, $2\left(\mathrm{VI}^{\mathrm{P}}\right)$. Le dém. lbs et le copte labes (S) qu'offre, en

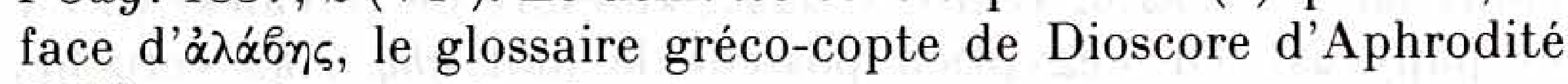
$\left(\mathrm{VI}^{\mathrm{P}}\right)$ paraissent découler du grec.

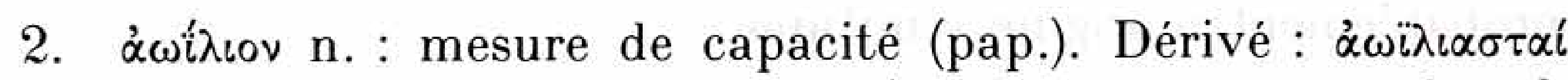
«terrassiers» (PCair. Zen. 59745, 58). On peut penser à aho, pl. ahōr (B), de l'ég. ' $h$ " $^{2}$ tas» (Wb I 220, 10-221, 1), mais ce serait peu satisfaisant.

67. Cf. Sethe "Der Name des Phönix", ZÄS 45 (1908), p. 85-9 suivi par Spiegelberg, ZÄS 46 (1909) p. 142, B. van de Walle et J. Vergote, Chronique d'Égypte, 18 (1943), p. 67 et J. Ch. Billigmeier, Talanta, 1977 p. 1-4, qui met en parallèle la ressemblance entre l'ég. bnw "phénix" et bny.t "palmier-dattier" avec

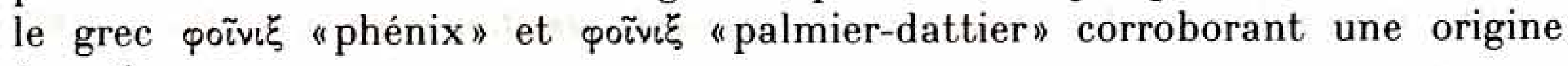
égyptienne.

68. PSI 428, $21 ; 862,11$. 


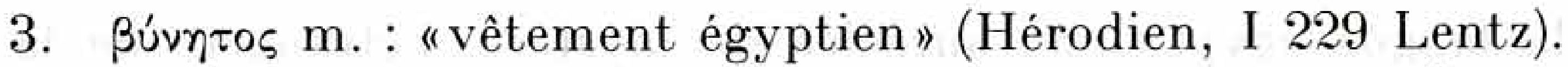
Ce mot isolé a-t-il un rapport avec l'ég. bnd "habiller» (Wb I $465,3)$ ?

4. vaúbı de terrassement) attestée dans les papyrus dès le III ${ }^{\text {a }}$ (PPetr. III 290, Ostr. Bodl. I 244, etc.), puis taxe foncière (PTeb. 5, 15; 76,$8 ; 119,52$ du II ${ }^{\text {a }}$, etc.). Wilcken (Ostr. I, p. 259 sq.) propose l'ég. $n b$ "panier». Peut-être faut-il plutôt rapprocher ce terme grec du copte nauben "pas» (Crum 235b)?

5. $\varphi \omega \sigma \sigma \omega \nu$ m. : "grosse étoffe de lin, vêtement de toile, bande de toile, voile, etc." (Lycophron, 26, la Souda; Eustathe,

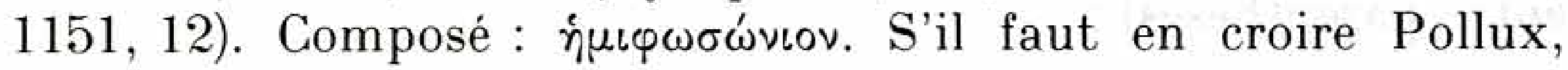
VII 71, il s'agirait d'un $\chi \iota \tau \omega \dot{\nu}$ égyptien. Demeure obscur.

Il nous faut ajouter en dernier lieu cinq hapax présents chez Hésychius :

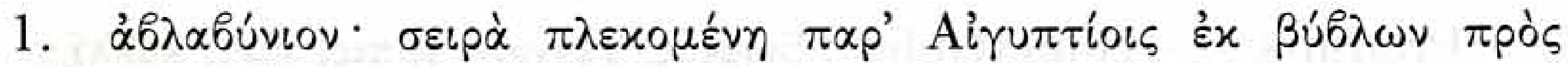

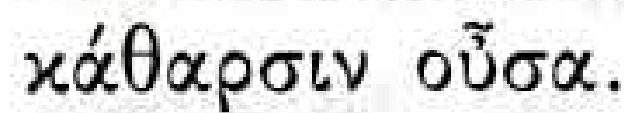

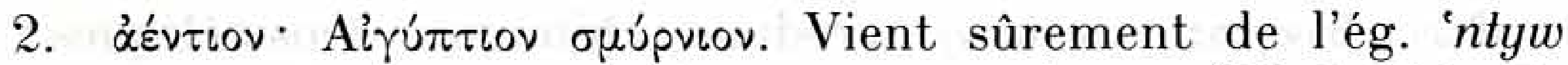
"gomme-résine, myrrhe, gomme aromatique» ( $W b$ I 206, 7 $297,3)$.

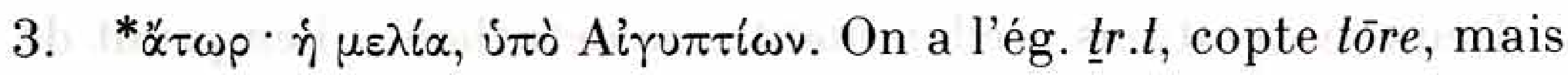
qui signifie "saule» et non "frêne".

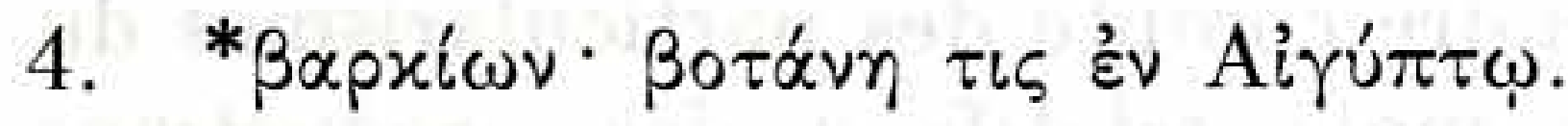

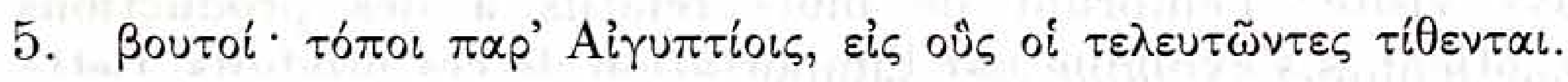

II. Essai d'interprétation

La première constatation qui s'impose, à la vue de cette liste, même partielle, est la très faible présence de mots égyptiens dans le lexique grec. Cela s'explique à la fois par la répugnance qu'a le grec à emprunter (préférant le recours à des équivalents, l'interpretatio graeca $^{69}$ ) et par les trois obstacles que constituent

69. Dans le domaine religieux : Athéna $=$ Neith, Héphaïstos $=$ Ptah. Dans le domaine géographique, cf. les noms de Troie $(=\operatorname{Tr} 3 w)$, d'Abydos $(=3 b d w)$; ceux d'Éphèse, Chios, Lesbos, Chypre, Samos donnés à des îles du Nil (Hécatée de Milet, Frg. 310 Jacoby). 
la langue égyptienne, auréolée de mystère, l'étrangeté de la civilisation conduisant soit à la mécompréhension soit à un réflexe d'auto-conservation culturelle (phénomène illustré avec éclat à l'époque ptolémaïque) et enfin l'éloignement géographique, qui explique l'absence quasi totale d'emprunts anciens. Les mots égyptiens dans la langue grecque sont rares, ils n'en sont pas moins révélateurs.

\section{Les motivations de l'emprunt.}

Les mots parfaitement assimilés dans la littérature grecque se répartissent en trois catégories (les emprunts probables sont entre parenthèses) :

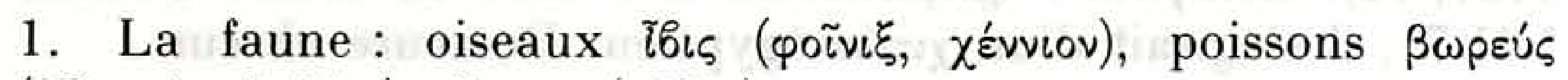

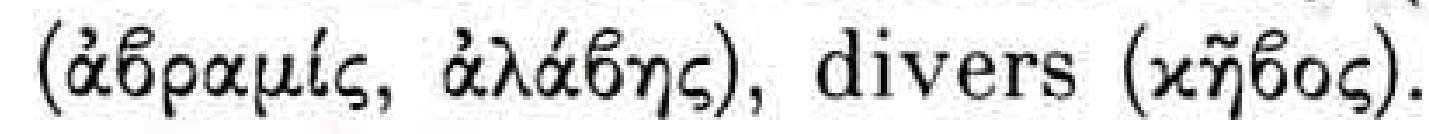

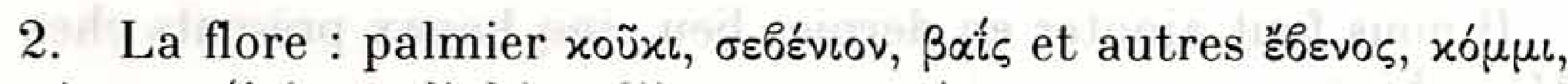
$\pi \alpha \dot{\pi}$ upos (faisant l'objet d'importation).

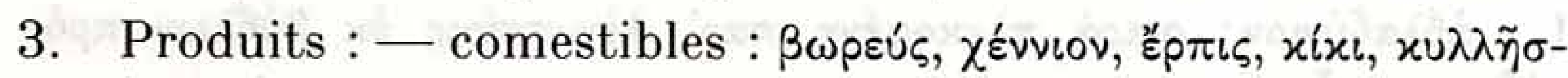
$\tau \iota \varsigma(\dot{\alpha} \theta \dot{\alpha} p \eta) ;-$ non-comestibles : vêtements, tissus $x \alpha \lambda \dot{\alpha} \sigma \iota p \iota \varsigma$

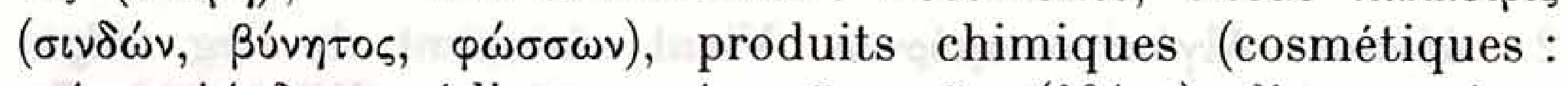

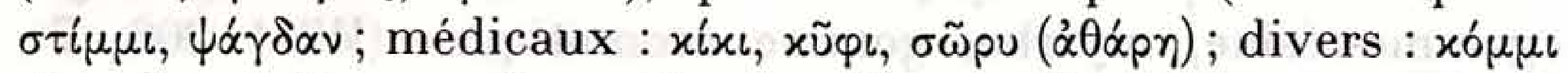

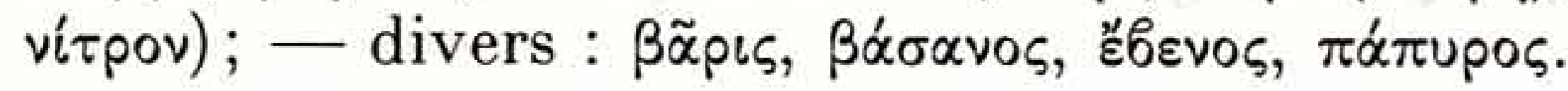

Deux motivations prédominantes se détachent : l'emprunt de mots relatifs à la faune et à la flore se justifie par des raisons d'enquêteurs désireux de rendre compte des particularismes du pays visité; l'emprunt de mots relatifs à des productions égyptiennes s'explique par l'importation de ces produits. Cette dernière motivation est largement majoritaire : on emprunte pour nommer ce dont on manque.

On constate que la vision qu'avaient les Grecs de l'Égypte et ce que nous savons du commerce entre les deux pays sont répercutés avec fidélité par les catégories lexicales que définissent les emprunts répertoriés : l'Égypte, exportatrice de lin, réputée pour ses tissus, à laquelle on attribuait l'invention du métier à tisser ${ }^{70}$; l'Égypte terre de la médecine, connue depuis

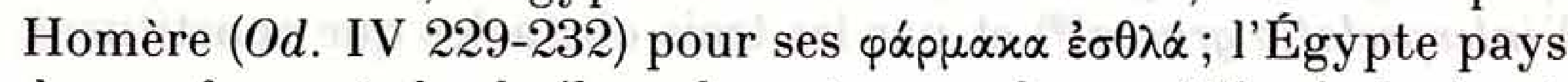
des parfums et des huiles odorantes que la comédie s'est plue à évoquer ... autant de visages qu'illustre notre liste sur le plan linguistique.

70. Eustathe, Ad Il. I 32. Athénée, II $48 \mathrm{c}$ nomme parmi trois célèbres tisseurs l'Égyptien Pathymias. 
Ainsi la plupart des emprunts du grec "métropolitain" s'expliquent par des relations commerciales très étroites et par la progressive apparition de produits égyptiens. Le recours à un mot étranger est nécessaire pour désigner un produit inconnu et parfois souhaitable pour des raisons de "marketing": le mot exotique rehausse le produit et lui confère l'attrait de la nouveauté.

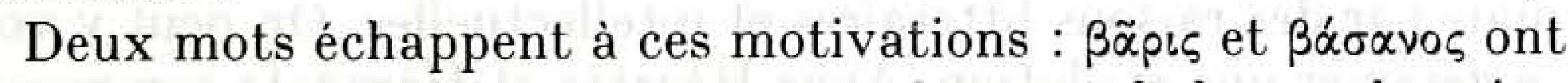
perdu, en tout cas totalement pour le second, leur coloration exotique. Ce sont les emprunts les plus remarquables.

Les emprunts du grec d'Égypte se répartissent en cinq catégories lexicales :

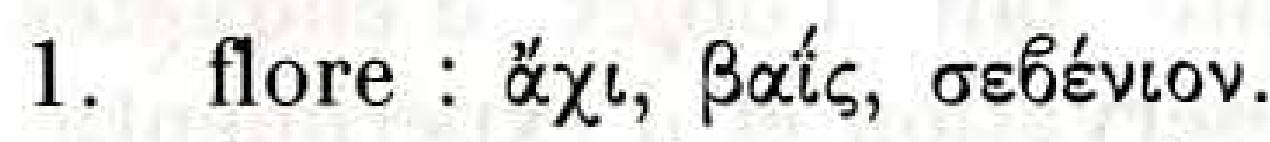

faune : xaíurov, xouxoúpas.

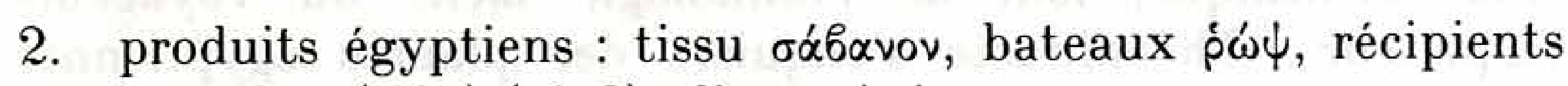

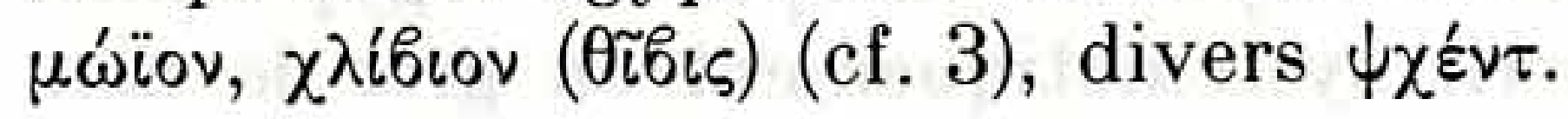

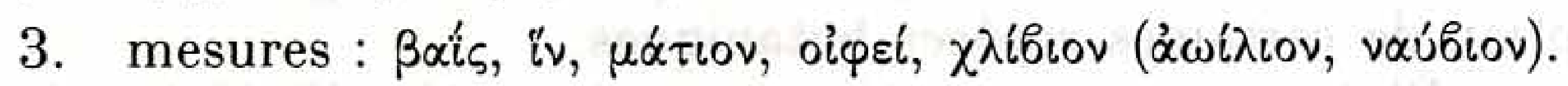

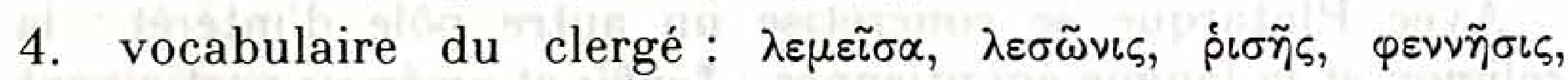
$\varphi \varepsilon v \pi \tau \alpha i ́ s$.

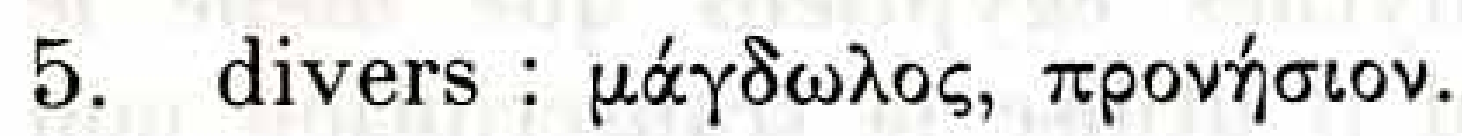

On peut s'étonner qu'une société grecque implantée en milieu égyptien n'ait emprunté que si peu de mots. Aussi ces derniers sont-ils pareillement motivés par la nécessité. Cependant il ne s'agit plus de dénommer des produits d'importation mais des éléments de la vie quotidienne. Un nouveau paysage se dessine : la présence de termes liés à la navigation démontre l'importance économique du Nil. Les éléments botaniques renvoient principalement au palmier, arbre de première importance, tandis qu' $\alpha$ ' $\chi$ b visualise un autre aspect de la flore égyptienne : ses étendues marécageuses en bordure du Nil et de ses bras.

L'innovation que révèle cet ensemble d'emprunts est le recours aux termes indigènes désignant des mesures et des fonctions cléricales. Tandis que l'adoption de termes métrologiques dénonce les échanges économiques entre Hellènes et Egyptiens, et la participation des premiers à la vie agricole du pays, l'utilisation du vocabulaire clérical égyptien démontre le souci qui fut celui des Lagides de préserver les particularismes religieux de l'Égypte, en plus de l'admiration respectueuse qu'ont toujours éprouvée les Grecs pour les temples de la vallée du Nil. C'est dans ce domaine que nous assistons à un 
phénomène unique : bien que le Grec répugne à emprunter, à plus forte raison s'il possède déjà un mot de même sens dans sa propre langue, il a cependant adopté le terme $\lambda \varepsilon \sigma \tilde{\omega} v \iota \varsigma$ bien que

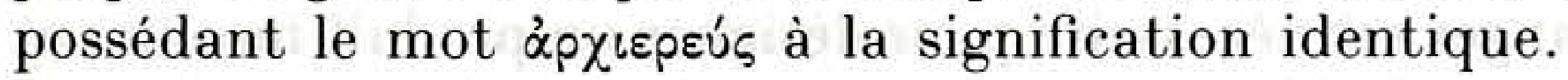

Avec les mots-transcriptions, nous entrons dans la catégorie des "emprunts de luxe», non motivés par la nécessité pratique, mais par des raisons littéraires et intellectuelles. On peut y voir se dessiner une évolution. Avec Hécatée et Hérodote commence la découverte de l'Égypte. Outre le vernis et le sérieux qu'il apporte à l'ouvrage, l'emprunt démontre surtout cet étonnement devant un monde inconnu et différent, au paysage inhabituel ('O $\left.{ }^{\prime} \sigma \iota \varsigma\right)$, à la faune curieuse $\left(\chi \alpha \dot{\alpha} \mu \psi_{\alpha \iota}\right)$. S'ouvre ensuite une deuxième période où l'Égypte fait l'objet d'enquêtes spécialisées, devient un champ d'observation et d'expérimentation scientifique, loin de l'ethnologie facile du voyageurenquêteur: Théophraste s'enquiert des plantes égyptiennes $(\sigma \alpha \dot{\rho} \iota)$. Dioscoride transcrit systématiquement en grec le nom égyptien de certaines espèces botaniques.

Avec Plutarque se concrétise un autre pôle d'intérêt: la religion et la langue égyptiennes: ${ }^{*} \mu \eta \theta i$ is et $\chi \varepsilon v o ́ \sigma \iota p \iota s ~ p a r t i c i p e n t$ de cette étude précise des mythes égyptiens que mène le philosophe de Chéronée, quand il utilise la transcription non pour dénommer une chose inconnue mais pour éclairer la signification profonde de cette chose dans sa relation avec des phénomènes religieux ou plus largement mystiques, tendance radicalisée par le hiérogrammate Horapollon $\left(V^{\mathrm{p}}\right)$.

Les emprunts des Septante sont justifiés par le mot hébreu qu'ils traduisent. Ils ont en effet eu recours à des vocables égyptiens probablement déjà passés dans la langue grecque presque courante, pour rendre des termes sémitiques de même

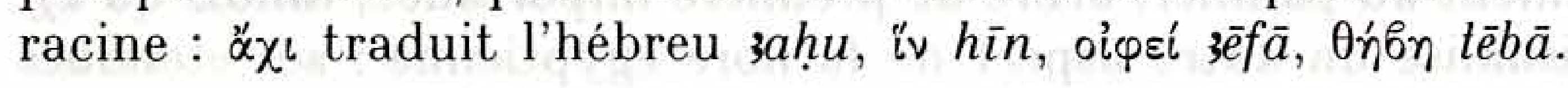

\section{Les modalités de l'emprunt.}

\section{A. Processus d'introduction.}

L'emprunt doit passer par une phase d'acquisition progressive qui implique des intermédiaires internes, à savoir individus ou groupes d'individus qui en répandent l'usage. Trois grandes voies ont été suivies par ces mots :

(1) la voie commerciale qui suppose un produit échangé dont on emprunte le nom, des comptoirs grecs en Égypte (donc des 
contacts entre populations différentes ${ }^{71}$ ) et la présence d'Égyptiens en Grèce (cible de moqueries de la part des Grecs qui ont pu les caractériser ou les stigmatiser par l'utilisation railleuse de quelques mots de leur sabir. Cf. l'emploi dans la comédie de $x \alpha \lambda \dot{\alpha} \sigma \iota p \iota \varsigma, x \cup \lambda \lambda \tilde{\eta} \sigma \tau \iota \zeta, \psi \dot{\alpha} \gamma \delta \alpha \nu$, etc.);

(2) les voies touristique et scientifique illustrées par les mots rares qu'ont contribué à diffuser Hécatée et Hérodote (i⿱宀ıı, etc.);

(3) la voie colonisatrice responsable de la présence de termes techniques dans les papyrus documentaires, termes qui ont parfois été réinjectés dans la xovv' à une époque tardive : $\sigma \alpha \dot{\alpha} b \alpha v o v$ se retrouve chez Clément d'Alexandrie, chez les médecins et

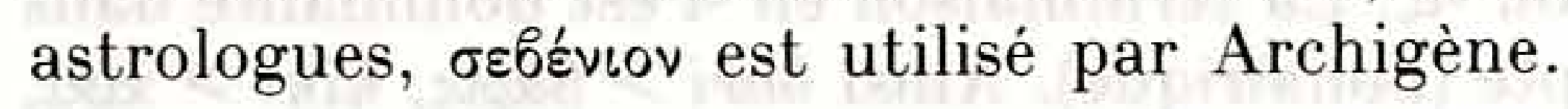

\section{B. Processus d'adaptation.}

L'adaptation phonétique implique trois paramètres qui justifient la plus ou moins grande distorsion ou variation du mot grec par rapport à son modèle :

- La difficulté des phonèmes égyptiens imprononçables pour un Hellène ou impossibles à rendre par un graphème grec. Le $h$ dont l'égyptien possède quatre variétés très différentes, en est une bonne illustration :

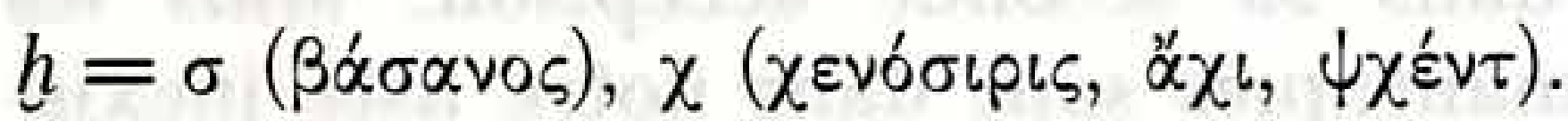

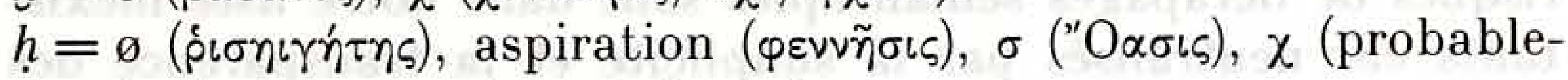
ment $\chi \dot{\alpha} \mu \psi \alpha_{\alpha}$, assurément $\left.\alpha^{\prime} \sigma \mu \alpha \chi\right)$.

- La nature des intermédiaires: l'altération est plus ou moins grande selon que le mot a suivi la voie populaire ou savante. C'est le paramètre le moins bien analysable car il laisse une grande part à l'individualité de l'intermédiaire.

- L'époque de l'emprunt: la langue égyptienne a vu sa prononciation évoluer. De plus les lettres grecques ont connu des valeurs phonétiques différentes au cours des temps. Le paramètre diachronique trouve une illustration dans les différentes tentatives pour rendre le $|\bar{o}|$ :

ex : III $^{\mathrm{e}}$ s. av. $p a-p \bar{o} r>\pi \dot{\alpha} \pi$ upos.

$\mathrm{I}^{\mathrm{er}}$ s. ap. bōre $>\beta \omega p \varepsilon u ́ c$.

Dans le premier temps (jusqu'au $\mathrm{II}^{\mathrm{e}} \mathrm{s}$. av.), le /ó:/ est rendu par le $u$. Le $\omega$ qui était à cette époque un $/ \dot{o}: /$ ne convenait pas

71. D'ailleurs le fait que les comptoirs grecs se situaient tous dans le Delta (Daphné, Naucratis) s'accorde bien avec la forme majoritairement bohaïrique des emprunts. 
pour transcrire le /ó:/, l'aperture primant sur la quantité (cf. $\mu \omega ́$ ïov qui transcrit ce qui va devenir en copte moeih). A partir du II $^{\mathrm{e}} \mathrm{s}$., le $\omega$ devient un $/ o ́$ : / et au moment de la création de l'alphabet copte, il est adopté en copte pour transcrire l'ég. |ó:/ tandis que le ou cette fois correspond au $\mid u /$, le $v$ grec étant devenu inapte à rendre cette valeur phonétique. C'est ainsi que

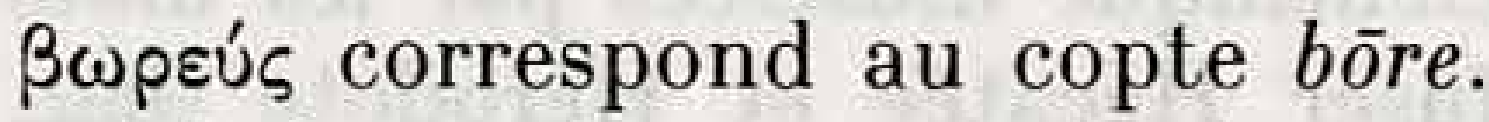

L'adaptation morphologique a permis aux mots empruntés de s'intégrer au prix d'un léger aménagement de la finale dans un groupe fonctionnel (ex. : $\beta \omega \rho \varepsilon u ́ \varsigma, ~ \rho ́ \sigma \tilde{\varsigma} \varsigma)$. De plus les terminaisons respectent souvent le vocalisme originel du mot : bōre $>\beta \omega p \varepsilon u ́ s$, *psagdan $>\psi \alpha \dot{\gamma} \gamma \alpha \varsigma$, thēbi $>0 \tilde{\imath} b \iota \varsigma$. Cela explique la grande majorité de noms en $-\iota$ ou $-\iota \varsigma$. La terminaison en $-\iota$ est dominante dans le nord de l'Égypte (bohaïrique). Ainsi bari $>\beta \tilde{\alpha} \rho\llcorner s, k i k i>x i ́ x \iota$, bai $>\beta \alpha i$ is, etc. Tant et si bien que le grammairien Théodore $\mathrm{H}$. consacre tout un paragraphe à ce qu'il appelle $\tau \dot{\alpha}$ sic $\overline{i \zeta}$ óvó $\mu \alpha \tau \alpha$ Airú $\tau \tau \iota \alpha$ (éd. Hildgard p. 196).

L'adaptation sémantique entre aussi en ligne de compte. Le mot étant déterminé, conditionné par son milieu, une fois transféré dans un autre contexte, il peut voir son sens originel modifié ou simplifié. Le mot xụ̃ı qui désignait en égyptien à la fois un encens cultuel et une fumigation médicale a été emprunté uniquement dans sa seconde acception. Mais les risques de dérapages sémantiques sont dans notre liste inexistants car neutralisés par la simplicité et la transparence des signifiés recouvrant des choses extrêmement concrètes.

\section{Conclusion}

L'étude des emprunts grecs à l'égyptien apporte un double témoignage : elle offre un champ d'investigation ouvert sur la meilleure compréhension du phénomène général qu'est l'emprunt linguistique, particulièrement sur les réflexes quasi inconscients de la langue emprunteuse. De plus elle jette une lumière nouvelle sur les rapports qui à travers les langues unissent deux civilisations, deux mentalités. Nous avons pu entrevoir que les renseignements linguistiques ainsi livrés sont en parfaite conjonction avec les données historiques et littéraires, y apportant bien souvent une touche de subjectivité que l'histoire, figée dans l'anecdotique et l'événementiel, ne laisse pas transparaître. 